\title{
ASYMPTOTIC STRUCTURE AND COARSE LIPSCHITZ GEOMETRY OF BANACH SPACES.
}

\author{
B. M. BRAGA
}

\begin{abstract}
In this paper, we study the coarse Lipschitz geometry of Banach spaces with several asymptotic properties. Specifically, we look at asymptotic uniform smoothness and convexity, and several distinct Banach-Saks-like properties. Among other results, we characterize the Banach spaces which are either coarsely or uniformly homeomorphic to $T^{p_{1}} \oplus \ldots \oplus T^{p_{n}}$, where each $T^{p_{j}}$ denotes the $p_{j}$-convexification of the Tsirelson space, for $p_{1}, \ldots, p_{n} \in(1, \ldots, \infty)$, and $2 \notin\left\{p_{1}, \ldots, p_{n}\right\}$. We obtain applications to the coarse Lipschitz geometry of the $p$-convexifications of the Schlumprecht space, and some hereditarily indecomposable Banach spaces. We also obtain some new results on the linear theory of Banach spaces.
\end{abstract}

\section{InTRODUCTION.}

In this paper, we study nonlinear embeddings and nonlinear equivalences between Banach spaces. For that, we look at a Banach space $(X,\|\cdot\|)$ as a metric space endowed with the metric $\|\cdot-\cdot\|$. Let $(M, d)$ and $(N, \partial)$ be metric spaces, and $f: M \rightarrow N$ be a map. For each $t \in[0, \infty)$, we define the expansion modulus of $f$ as

$$
\omega_{f}(t)=\sup \{\partial(f(x), f(y)) \mid d(x, y) \leq t\}
$$

and the compression modulus of $f$ as

$$
\rho_{f}(t)=\inf \{\partial(f(x), f(y)) \mid d(x, y) \geq t\}
$$

We say that $f$ is a coarse map if $\omega_{f}(t)<\infty$, for all $t \in[0, \infty)$. If, in addition, $\lim _{t \rightarrow \infty} \rho_{f}(t)=\infty$, then $f$ is a coarse embedding. We say that $f$ is a coarse equivalence if $f$ is both a coarse embedding and cobounded, i.e., $\sup _{y \in N} \partial(y, f(M))<\infty$. The map $f$ is a uniform embedding if $\lim _{t \rightarrow 0_{+}} \omega_{f}(t)=0$ and $\rho_{f}(t)>0$, for all $t \in(0, \infty)$. A surjective uniform embedding is called a uniform homeomorphism. If there exists $L>0$ such that $\omega_{f}(t) \leq L t+L$, for all $t \in[0, \infty)$, then we call $f$ a coarse Lipschitz map. If, in addition, $\rho_{f}(t) \geq L^{-1} t-L$, for all $t \in[0, \infty)$, then $f$ is a coarse Lipschitz embedding.

A uniformly continuous map $f: X \rightarrow N$ from a Banach space $X$ to a metric space $N$ is automatically a coarse map (see $[\underline{\mathrm{K}}$, Lemma 1.4). Similarly, $f: X \rightarrow M$ is a coarse map if and only if it is a coarse Lipschitz map (see $[\mathrm{K}$, Lemma 1.4).

2010 Mathematics Subject Classification. Primary: 46B80.

Key words and phrases. 
Also, if two Banach spaces $X$ and $Y$ are coarsely equivalent (resp. uniformly homeomorphic) then $X$ coarse Lipschitz embeds into $Y$ (see $[\mathbf{K}]$, Proposition 1.5).

In these notes, we are mainly interested in what kind of stability properties those notions of nonlinear embeddings and nonlinear equivalences may have, and we will mainly work with Banach spaces having some kind of asymptotic property. More specifically, we are concerned with asymptotically uniformly smooth Banach spaces, asymptotically uniformly convex Banach spaces, and Banach spaces having several different Banach-Saks-like properties (we refer to Section 2 for precise definitions).

The following general question is a central problem when dealing with nonlinear embeddings between Banach spaces.

Problem 1.1. Let $\mathcal{P}$ and $\mathcal{P}^{\prime}$ be two classes of Banach spaces and $\mathcal{E}$ be a kind of nonlinear embedding between Banach spaces. If a Banach space $X \mathcal{E}$-embeds into a Banach space $Y$ in $\mathcal{P}$, does it follow that $X$ is in $\mathcal{P}^{\prime}$ ?

For example, if a separable Banach space $X$ coarse Lipschitz embeds into a superreflexive Banach space, then $X$ is also super-reflexive (this follows from Proposition 1.6 of $[\mathrm{K}]$ and Theorem 2.4 of $[\mathrm{K}$, but it was first proved for uniform equivalences in Ri], Theorem 1A). Another example was given by M. Mendel and A. Naor in $\mathrm{MeN}$ (Theorem 1.11), where they showed that if a Banach space $X$ coarsely embeds into a Banach space $Y$ with cotype $q$ and non trivial type, then $X$ has cotype $q+\varepsilon$, for all $\varepsilon>0$.

If we look at nonlinear equivalences between Banach spaces, the following is a central problem in the theory.

Problem 1.2. Let $X$ be a Banach space and $\mathcal{E}$ be a kind of nonlinear equivalence between Banach spaces. If a Banach space $Y$ is $\mathcal{E}$-equivalent to $X$, what can we say about the isomorphism type of $Y$ ? More precisely:

(i) Is the linear structure of $X$ determined by its $\mathcal{E}$-structure, i.e., if a Banach space $Y$ is $\mathcal{E}$-equivalent to $X$, does it follow that $Y$ is linearly isomorphic to $X$ ?

(ii) Let $\mathcal{P}$ be a class of Banach spaces. If $Y$ is $\mathcal{E}$-equivalent to $X$, does is follow that $Y$ is linearly isomorphic to $X \oplus Z$, for some Banach space $Z$ in $\mathcal{P}$ ?

Along those lines, it was shown in JoLiS (Theorem 2.1) that the coarse (resp. uniform) structure of $\ell_{p}$ completely determines its linear structure, for any $p \in$ $(1, \infty)$. For $p=1$, we do not even know if the Lipschitz structure of $\ell_{1}$ determines its linear structure. N. Kalton and N. Randrianarivony proved in KRa (Theorem $5.4)$ that, for any $p_{1}, \ldots, p_{n} \in(1, \infty)$ with $2 \notin\left\{p_{1}, \ldots, p_{n}\right\}$, the linear structure of $\ell_{p_{1}} \oplus \ldots \oplus \ell_{p_{n}}$ is determined by its coarse (resp. uniform) structure (see also JoLiS, Theorem 2.2). This problem is still open if $2 \in\left\{p_{1}, \ldots, p_{n}\right\}$.

Let $T$ denote the Tsirelson space introduced by T. Fiegel and W. Johnson in FJo. For each $p \in[1, \infty)$, let $T^{p}$ be the $p$-convexification of $T$ (see Subsection 2.6 for definitions). W. Johnson, J. Lindenstrauss and G. Schechtman addressed Problem 1.2(ii) above by proving the following (see JoLiS, Theorem 5.8): suppose that either $1<p_{1}<\ldots<p_{n}<2$ or $2<p_{1}<\ldots<p_{n}$ and set $X=T^{p_{1}} \oplus \ldots \oplus T^{p_{n}}$, then a Banach space $Y$ is coarsely equivalent (resp. uniformly homeomorphic) to $X$ if and only if $Y$ is linearly isomorphic to $X \oplus \bigoplus_{j \in F} \ell_{p_{j}}$, for some $F \subset\{1, \ldots, n\}$.

We now describe the organization and some of the results of this paper. Firstly, in order not to make this introduction too extensive, we will postpone some technical 
definitions for later as well as our more technical results. The reader will find all the background and notation necessary for this paper in Section 2 ,

Along the lines of Problem 1.1 we prove the following in Section 3 .

Theorem 1.3. Let $Y$ be a reflexive asymptotically uniformly smooth Banach space, and assume that a Banach space $X$ coarse Lipschitz embeds into $Y$. Then $X$ has the Banach-Saks property.

As the Banach-Saks property implies reflexivity, Theorem 1.3 above is a strengthening of Theorem 4.1 of BKL, where the authors showed that if a separable Banach space $X$ coarse Lipschitz embeds into a reflexive asymptotically uniformly smooth Banach space, then $X$ must be reflexive. As $T$ is a reflexive Banach space without the Banach-Saks property, Theorem 1.3 gives us the following new corollary.

Corollary 1.4. The Tsirelson space does not coarse Lipschitz embed into any reflexive asymptotically uniformly smooth Banach space.

In Section 3 we also prove some results on the linear theory of Banach spaces. Precisely, we show that an asymptotically uniformly smooth Banach space $X$ must have the alternating Banach-Saks property (see Corollary 3.2). Using descriptive set theoretical arguments, we also show that the converse does not hold, i.e., that there are Banach spaces with the alternating Banach-Saks property which do not admit an asymptotically uniformly smooth renorming (see Proposition 3.8).

In Section 4 we study coarse embeddings $f: X \rightarrow Y$ between Banach spaces $X$ and $Y$ with specific asymptotic properties, and obtain a general result on how close to an affine map the compression modulus $\rho_{f}$ can be (see Theorem 4.1). Precisely, E. Guentner and J. Kaminker introduced the following quantity in GuKa: for Banach spaces $X$ and $Y$, define $\alpha_{Y}(X)$ as the supremum of all $\alpha>0$ for which there exists a coarse embedding $f: X \rightarrow Y$ and $L>0$ such that $\rho_{f}(t) \geq L^{-1} t^{\alpha}-L$, for all $t \geq 0$. We call $\alpha_{Y}(X)$ the compression exponent of $X$ in $Y$. As a simple consequence of Theorem 4.1, we obtain Theorem 1.5 below.

We denote by $S$ the Schlumprecht space introduced in $\underline{\underline{S c}}$, and, for each $p \in$ $[1, \infty)$, we let $S^{p}$ be the $p$-convexification of $S$ and $T^{p}$ be the $p$-convexification of the Tsirelson space $T$ (see Subsection 2.6 for definitions).

Theorem 1.5. Let $1 \leq p<q$. Then

(i) $\alpha_{T^{q}}\left(T^{p}\right) \leq p / q$, and

(ii) $\alpha_{S^{q}}\left(S^{p}\right) \leq p / q$.

In particular, $T^{p}$ (resp. $\left.S^{p}\right)$ does not coarse Lipschitz embed into $T^{q}$ (resp. $\left.S^{q}\right)$.

The proof of Theorem 1.5 is asymptotically in nature, hence we obtain equivalent estimates for the compression exponent $\alpha_{Y}(X)$, where $X$ and $Y$ are Banach spaces satisfying some special asymptotic properties. In particular, the spaces $T^{q}$ and $S^{q}$ can be replaced in Theorem 1.5 by $\left(\oplus_{n} E_{n}\right)_{T^{q}}$ and $\left(\oplus_{n} E_{n}\right)_{S^{q}}$, where $\left(E_{n}\right)_{n=1}^{\infty}$ is any sequence of finite dimensional Banach spaces. See Theorem 4.3, Theorem 4.5 and Corollary 4.7 for precise statements.

We also apply our results to the hereditarily indecomposable Banach spaces $\mathfrak{X}^{p}$ defined by $\mathrm{N}$. Dew in $\left[\mathrm{D}\right.$, and obtain that $\alpha_{\mathfrak{X}^{q}}\left(\mathfrak{X}^{p}\right) \leq p / q$, for $1<p<q$ (see Corollary 4.8). 
In Section 5, we prove a general theorem regarding the non existence of coarse Lipschitz embeddings $X \rightarrow Y_{1} \oplus Y_{2}$, for Banach spaces $X, Y_{1}, Y_{2}$ with specific asymptotic properties (see Theorem [5.6). With that result in hands, we prove the following.

Theorem 1.6. Let $1 \leq p_{1}<\ldots<p_{n}<\infty$, and $p \in[1, \infty) \backslash\left\{p_{1}, \ldots, p_{n}\right\}$. Then neither $T^{p}$ nor $\ell_{p}$ coarse Lipschitz embed into $T^{p_{1}} \oplus \ldots \oplus T^{p_{n}}$. In particular, $T^{p}$ does not coarse Lipschitz embed into $T^{q}$, for all $p, q \in[1, \infty)$ with $p \neq q$.

At last, we use Theorem [1.6 in order to obtain the following characterization.

Theorem 1.7. Let $1<p_{1}<\ldots<p_{n}<\infty$ with $2 \notin\left\{p_{1}, \ldots, p_{n}\right\}$. A Banach space $Y$ is coarsely equivalent (resp. uniformly homeomorphic) to $X=T^{p_{1}} \oplus \ldots \oplus T^{p_{n}}$ if and only if $Y$ is linearly isomorphic to $X \oplus \bigoplus_{j \in F} \ell_{p_{j}}$, for some $F \subset\{1, \ldots, n\}$.

Clearly, Theorem [1.7 is a strengthening of Theorem 5.8 of JoLiS mentioned above. However, just as in the case for $\ell_{p_{1}} \oplus \ldots \oplus \ell_{p_{n}}$, we still do not know whether the theorem above holds if $2 \in\left\{p_{1}, \ldots, p_{n}\right\}$.

\section{Notation AND BACKGROUnd.}

2.1. Basic definitions. All the Banach spaces in these notes are assumed to be infinite dimensional unless otherwise stated. Let $X$ be a Banach space. We denote the closed unit ball of $X$ by $B_{X}$, and its unit sphere by $\partial B_{X}$. If $Y$ is also a Banach space, we write $X \cong Y$ if $X$ is linearly isomorphic to $Y$. Given a Banach space $X$ with norm $\|\cdot\|_{X}$, we simply write $\|\cdot\|$ as long as it is clear from the context to which space the elements inside the norm belong to. A sequence $\left(x_{n}\right)_{n=1}^{\infty}$ in a Banach space $X$ is called semi-normalized if it is bounded and $\inf _{n}\left\|x_{n}\right\|>0$.

Say $\left(e_{n}\right)_{n=1}^{\infty}$ is a basis for the Banach space $X$. For $x=\sum_{n=1}^{\infty} x_{n} e_{n} \in X$, we write $\operatorname{supp}(x)=\left\{n \in \mathbb{N} \mid x_{n} \neq 0\right\}$. For all finite subsets $E, F \subset \mathbb{N}$, we write $E<F$ (resp. $E \leq F$ ) if $\max E<\min F$ (resp. $\max E \leq \min F$ ). We call a sequence $\left(y_{n}\right)_{n=1}^{\infty}$ in $X$ a block sequence of $\left(e_{n}\right)_{n=1}^{\infty}$ if $\operatorname{supp}\left(y_{n}\right)<\operatorname{supp}\left(y_{n+1}\right)$, for all $n \in \mathbb{N}$.

Let $\left(X_{n}\right)_{n=1}^{\infty}$ be a sequence of Banach spaces. Let $\mathcal{E}=\left(e_{n}\right)_{n=1}^{\infty}$ be a 1-unconditional basic sequence in a Banach space $E$ with norm $\|\cdot\|_{E}$. We define the sum $\left(\oplus_{n} X_{n}\right)_{\mathcal{E}}$ to be the space of sequences $\left(x_{n}\right)_{n=1}^{\infty}$, where $x_{n} \in X_{n}$, for all $n \in \mathbb{N}$, such that

$$
\left\|\left(x_{n}\right)_{n=1}^{\infty}\right\|:=\left\|\sum_{n \in \mathbb{N}}\right\| x_{n}\left\|e_{n}\right\|_{E}<\infty .
$$

One can check that $\left(\oplus_{n} X_{n}\right)_{\mathcal{E}}$ endowed with the norm $\|\cdot\|$ defined above is a Banach space. If the $X_{n}$ 's are all the same, say $X_{n}=X$, for all $n \in \mathbb{N}$, we write $(\oplus X)_{\mathcal{E}}$. Also, if it is implicit what is the basis $\mathcal{E}$ of the Banach space $E$ that we are working with, we write $\left(\oplus_{n} X_{n}\right)_{E}$.

2.2. $p$-convex and $p$-concave Banach spaces. Let $X$ be a Banach space with 1-unconditional basis $\left(e_{n}\right)_{n=1}^{\infty}$, and let $p \in(1, \infty)$. We say that the basis $\left(e_{n}\right)_{n=1}^{\infty}$ is $p$-convex with convexity constant $C$ (resp. $p$-concave with concavity constant $C$ ), if

$$
\left\|\sum_{j \in \mathbb{N}}\left(\left|x_{j}^{1}\right|^{p}+\ldots+\left|x_{j}^{k}\right|^{p}\right)^{1 / p} e_{j}\right\|^{p} \leq C^{p} \sum_{n=1}^{k}\left\|x^{n}\right\|^{p},
$$




$$
\text { (resp. } \left.\quad C^{p}\left\|\sum_{j \in \mathbb{N}}\left(\left|x_{j}^{1}\right|^{p}+\ldots+\left|x_{j}^{k}\right|^{p}\right)^{1 / p} e_{j}\right\|^{p} \geq \sum_{n=1}^{k}\left\|x^{n}\right\|^{p}\right),
$$

for all $x^{1}=\sum_{j=1}^{\infty} x_{j}^{1} e_{j}, \ldots, x^{k}=\sum_{j=1}^{\infty} x_{j}^{k} e_{j} \in X$. We say that the basis $\left(e_{n}\right)_{n=1}^{\infty}$ satisfies an upper $\ell_{p}$-estimate with constant $C$ (resp. lower $\ell_{p}$-estimate with constant $C)$, if

$$
\left\|x_{1}+\ldots+x_{k}\right\|^{p} \leq C^{p} \sum_{n=1}^{k}\left\|x_{n}\right\|^{p} \quad\left(\operatorname{resp} . C^{p}\left\|x_{1}+\ldots+x_{k}\right\|^{p} \geq \sum_{n=1}^{k}\left\|x_{n}\right\|^{p}\right),
$$

for all $x_{1}, \ldots, x_{k} \in X$ with disjoint supports. Clearly, a $p$-convex (resp. $p$-concave) basis with constant $C$ satisfies an upper (resp. lower) $\ell_{p}$-estimate with constant $C$.

2.3. $p$-convexification. Let $X$ be a Banach space with a 1-unconditional basis $\left(e_{n}\right)_{n=1}^{\infty}$. For any $p \in[1, \infty)$, we define the $p$-convexification of $X$ as follows. Let

$$
X^{p}=\left\{\left.\left(x_{n}\right)_{n=1}^{\infty} \in \mathbb{R}^{\mathbb{N}}\left|x^{p}:=\sum_{n \in \mathbb{N}}\right| x_{n}\right|^{p} e_{n} \in X\right\}
$$

and endow $X^{p}$ with the norm $\|x\|_{p}=\left\|x^{p}\right\|^{1 / p}$, for all $x \in X^{p}$. By abuse of notation, we denote by $\left(e_{n}\right)_{n=1}^{\infty}$ the sequence of coordinate vectors in $X^{p}$. It is clear that $\left(e_{n}\right)_{n=1}^{\infty}$ is a 1-unconditional basis for $X^{p}$ and that $X^{1}=X$. Also, the triangle inequality gives us that $X^{p}$ is $p$-convex with constant 1 .

2.4. Asymptotically $p$-uniformly smooth and convex spaces. Let $X$ be a Banach space. We define the modulus of asymptotic uniform smoothness of $X$ as

$$
\bar{\rho}_{X}(t)=\sup _{x \in \partial B_{X}} \inf _{\operatorname{dim}(X / E)<\infty} \sup _{h \in \partial B_{E}}\|x+t h\|-1 .
$$

We say that $X$ is asymptotically uniformly smooth if $\lim _{t \rightarrow 0_{+}} \bar{\rho}_{X}(t) / t=0$. If there exists $p \in(1, \infty)$ and $C>0$ such that $\bar{\rho}_{X}(t) \leq C t^{p}$, for all $t \in[0,1]$, we say that $X$ is asymptotically p-uniformly smooth. Every asymptotically uniformly smooth Banach space is asymptotically $p$-uniformly smooth for some $p \in(1, \infty)$ (this was first proved in KnOSh for separable Banach spaces, and later generalized for any Banach space in $[\mathrm{R}$, Theorem 1.2).

Let $X$ be a Banach space. We define the modulus of asymptotic uniform convexity of $X$ as

$$
\bar{\delta}_{X}(t)=\inf _{x \in \partial B_{X}} \sup _{\operatorname{dim}(X / E)<\infty} \inf _{h \in \partial B_{E}}\|x+t h\|-1 .
$$

We say that $X$ is asymptotically uniformly convex if $\bar{\delta}_{X}(t)>0$, for all $t>0$. If there exists $p \in(1, \infty)$ and $C>0$ such that $\bar{\delta}_{X}(t) \geq C t^{p}$, for all $t \in[0,1]$, we say that $X$ is asymptotically p-uniformly convex.

The following proposition is straight forward.

Proposition 2.1. Let $p \in(1, \infty)$ and let $X$ be a Banach space with a 1-unconditional basis satisfying an upper $\ell_{p}$-estimate (resp. lower $\ell_{p}$-estimate) with constant 1. Then $X$ is asymptotically p-uniformly smooth (resp. asymptotically p-uniformly convex). 
2.5. Banach-Saks properties. A Banach space $X$ is said to have the BanachSaks property if every bounded sequence $\left(x_{n}\right)_{n=1}^{\infty}$ in $X$ has a subsequence $\left(x_{n_{j}}\right)_{j=1}^{\infty}$ such that $\left(\frac{1}{k} \sum_{j=1}^{k} x_{n_{j}}\right)_{k=1}^{\infty}$ converges. A Banach space $X$ is said to have the alternating Banach-Saks property if every bounded sequence $\left(x_{n}\right)_{n=1}^{\infty}$ in $X$ has a subsequence $\left(x_{n_{j}}\right)_{j=1}^{\infty}$ such that $\left(\frac{1}{k} \sum_{j=1}^{k} \varepsilon_{j} x_{n_{j}}\right)_{k=1}^{\infty}$ converges, for some $\left(\varepsilon_{j}\right)_{j=1}^{\infty} \in$ $\{-1,1\}^{\mathbb{N}}$. For a detailed study of this properties, we refer to $[\mathrm{Be}$.

Let $p \in(1, \infty)$. A Banach space $X$ is said to have the $p$-Banach-Saks property (resp. p-co-Banach-Saks property), if for every semi-normalized weakly null sequence $\left(x_{n}\right)_{n=1}^{\infty}$ in $X$, there exists a subsequence $\left(x_{n_{j}}\right)_{j=1}^{\infty}$ and $c>0$ such that

$$
\left\|x_{n_{1}}+\ldots+x_{n_{k}}\right\| \leq c k^{1 / p} \quad\left(\text { resp. } \quad\left\|x_{n_{1}}+\ldots+x_{n_{k}}\right\| \geq c k^{1 / p}\right),
$$

for all $k \in \mathbb{N}$, and all $k \leq n_{1}<\ldots<n_{k}$.

The following is a combination of Proposition 1.2, Proposition 1.3, and Proposition 1.6 of DimGoJ] (Proposition 1.6 of DimGoJ only mentions the $p$-Banach-Saks property, but a straight forward modification of their proof gives us the result for the $p$-co-Banach-Saks property).

Proposition 2.2. Let $p \in(1, \infty)$ and let $X$ be a Banach space. If $X$ asymptotically $p$-uniformly smooth (resp. asymptotically p-uniformly convex), then $X$ has the $p$ Banach-Saks property (resp. p-co-Banach-Saks property)

2.6. Tsirelson and Schlumprecht spaces. Let $c_{00}$ denote the set of sequences of real numbers which are eventually zero, and let $\|\cdot\|_{0}$ be the max norm on $c_{00}$. We denote by $T$ the Tsirelson space defined in [FJo, i.e., $T$ is the completion of $c_{00}$ under the unique norm $\|\cdot\|$ satisfying

$$
\|x\|=\max \left\{\|x\|_{0}, \frac{1}{2} \cdot \sup \left(\sum_{j=1}^{k}\left\|E_{j} x\right\|\right)\right\},
$$

where the inner supremum above is taken over all finite sequences $\left(E_{j}\right)_{j=1}^{k}$ of finite subsets of $\mathbb{N}$ such that $k \leq E_{1}<\ldots<E_{k}$. Therefore, for each $p \in(1, \infty)$, the norm $\|\cdot\|_{p}$ of the $p$-convexified Tsirelson space $T^{p}$ satisfies

$$
\|x\|_{p}=\max \left\{\|x\|_{0}, \frac{1}{2^{1 / p}} \cdot \sup \left(\sum_{j=1}^{k}\left\|E_{j} x\right\|_{p}^{p}\right)^{1 / p}\right\},
$$

where the inner supremum above is taken over all finite sequences $\left(E_{j}\right)_{j=1}^{k}$ of finite subsets of $\mathbb{N}$ such that $k \leq E_{1}<\ldots<E_{k}$ (see [CSh, Chapter X, Section E).

As $T^{p}$ satisfies an upper $\ell_{p}$-estimate with constant 1 , it follows that $T^{p}$ is asymptotically $p$-uniformly smooth and it has the $p$-Banach-Saks property. Also, $T^{p}$ has the $p$-co-Banach-Saks property. Indeed, let $\left(e_{n}\right)_{n=1}^{\infty}$ be the standard basis for $T^{p}$. If $\left(x_{n}\right)_{n=1}^{\infty}$ is a normalized block subsequence of $\left(e_{n}\right)_{n=1}^{\infty}$, then

$$
2^{-1 / p} k^{1 / p}=2^{-1 / p}\left(\sum_{n=k}^{2 k-1}\left\|x_{n}\right\|_{p}^{p}\right)^{1 / p} \leq\left\|\sum_{n=k}^{2 k-1} x_{n}\right\|_{p},
$$


for all $k \in \mathbb{N}$. Therefore, as for any normalized weakly null sequence $\left(x_{n}\right)_{n=1}^{\infty}$ in $T^{p}$, one can find a block sequence $\left(y_{n}\right)_{n=1}^{\infty}$ which is equivalent to a subsequence of $\left(x_{n}\right)_{n=1}^{\infty}$, we conclude that $T^{p}$ has the $p$-co-Banach-Saks.

Remark 2.3. Let $p \in(1, \infty)$. Then $T^{p}$ does not contain $\ell_{r}$ for any $r \in[1, \infty)$ (this is shown in Jo2 for $T$, and the result for $T^{p}$ follows analogously). Similarly, by duality arguments, $T^{p *}$ does not contain $\ell_{r}$ for any $r \in[1, \infty)$ (the reader can find more on $T^{p}$ and similar duality arguments in [CSh]).

The Schlumprecht space $S$ (see $\left[\mathrm{Sc}\right.$ ) is defined as the completion of $c_{00}$ under the unique norm $\|\cdot\|$ satisfying

$$
\|x\|=\max \left\{\|x\|_{0}, \sup \left(\frac{1}{\log _{2}(k+1)} \sum_{j=1}^{k}\left\|E_{j} x\right\|\right)\right\},
$$

where the inner supremum above is taken over all finite sequences $\left(E_{j}\right)_{j=1}^{k}$ of finite subsets of $\mathbb{N}$ such that $E_{1}<\ldots<E_{k}$. Similarly as with the $p$-convexified Tsirelson space, the norm $\|\cdot\|_{p}$ of the $p$-convexified Schlumprecht space $S^{p}$ is given by

$$
\|x\|_{p}=\max \left\{\|x\|_{0}, \sup \left(\frac{1}{\log _{2}(k+1)} \sum_{j=1}^{k}\left\|E_{j} x\right\|_{p}^{p}\right)^{1 / p}\right\},
$$

where the inner supremum above is taken over all finite sequences $\left(E_{j}\right)_{j=1}^{k}$ of finite subsets of $\mathbb{N}$ such that $E_{1}<\ldots<E_{k}$ (see [D], page 59).

Similarly to $T^{p}, S^{p}$ is asymptotically $p$-uniformly smooth and has the $p$-BanachSaks property, for $p \in(1, \infty)$.

2.7. Almost $p$-co-Banach-Saks property. Although $T^{p}$ has the $p$-co-BanachSaks property, $S^{p}$ does not. However, $S^{p}$ satisfies a weaker property that will be enough for our goals. Let $p \in(1, \infty)$. We say that a Banach space $X$ has the $a$ most p-co-Banach-Saks property if for every semi-normalized weakly null sequence $\left(x_{n}\right)_{n=1}^{\infty}$ in $X$ there exists a subsequence $\left(x_{n_{j}}\right)_{j=1}^{\infty}$, and a sequence of positive numbers $\left(\theta_{j}\right)_{j=1}^{\infty}$ in $[1, \infty)$ such that $\lim _{j \rightarrow \infty} j^{\alpha} \theta_{j}^{-1}=\infty$, for all $\alpha>0$, and

$$
\left\|x_{n_{1}}+\ldots+x_{n_{k}}\right\| \geq k^{1 / p} \theta_{k}^{-1},
$$

for all $k \in \mathbb{N}$, and all $k \leq n_{1}<\ldots<n_{k}$. Clearly, $S^{p}$ has the almost $p$-co-BanachSaks property, with $\theta_{k}=\log _{2}(k+1)^{1 / p}$, for all $k \in \mathbb{N}$.

\section{Asymptotic Uniform smoothness and the alternating Banach-SaKs PROPERTY.}

In this section, we are going to show that asymptotically uniformly smooth Banach spaces must have the alternating Banach-Saks property (Corollary 3.2), but the converse does not hold (see Proposition 3.8). Also, we show that if a Banach space $X$ coarse Lipschitz embeds into a reflexive space $Y$ which is also asymptotically uniformly smooth, then $X$ must have the Banach-Saks property (Theorem 1.3). As any space with the Banach-Saks property is reflexive, this is a strengthening of Theorem 4.1 of BKL, which says that, under the same hypothesis, $X$ must be reflexive. 
Proposition 3.1. Let $X$ be a Banach space with the p-Banach-Saks property, for some $p \in(1, \infty)$, and assume that $X$ does not contain $\ell_{1}$. Then $X$ has the alternating Banach-Saks property. In particular, if $X$ is also reflexive, then $X$ has the Banach-Saks property.

Proof. Assume $X$ does not have the alternating Banach-Saks property. Then, there exist $\delta>0$ and a bounded sequence $\left(x_{n}\right)_{n=1}^{\infty}$ in $X$ such that, for all $k \in \mathbb{N}$, all $\varepsilon_{1}, \ldots, \varepsilon_{k} \in\{-1,1\}$, and all $n_{1}<\ldots<n_{k} \in \mathbb{N}$, we have

$$
\left\|\frac{1}{k} \sum_{j=1}^{k} \varepsilon_{j} x_{n_{j}}\right\|>\delta
$$

(see [Be, Theorem 1, page 369). As $X$ does not contain $\ell_{1}$, by Rosenthal's $\ell_{1}$ theorem (see [Ro]), we can assume that $\left(x_{n}\right)_{n=1}^{\infty}$ is weakly Cauchy. Hence, the sequence $\left(x_{2 n-1}-x_{2 n}\right)_{n=1}^{\infty}$ is weakly null. By Equation (3.1), it is also seminormalized. Therefore, as $X$ has the $p$-Banach-Saks property, by taking a subsequence if necessary, we have that

$$
\left\|\sum_{j=1}^{k}\left(x_{n_{2 j-1}}-x_{n_{2 j}}\right)\right\| \leq c k^{1 / p},
$$

for all $k \in \mathbb{N}$, and some constant $c>0$ independent of $k$. By Equation (3.1), we get that

$$
\delta<\left\|\frac{1}{2 k} \sum_{j=1}^{2 k}(-1)^{j+1} x_{n_{j}}\right\| \leq \frac{c}{2} k^{1 / p-1} .
$$

As this holds for all $k \in \mathbb{N}$, and $p>1$, if we let $k \rightarrow \infty$, we get that $\delta=0$, which is a contradiction.

For reflexive spaces, the alternating Banach-Saks property and the Banach-Saks property are equivalent (see $[\mathrm{Be}$, Proposition 2), so the last statement of the proposition follows.

Corollary 3.2. Let $X$ be an asymptotically uniformly smooth Banach space. Then $X$ has the alternating Banach-Saks property. In particular, if $X$ is also reflexive, then $X$ has the Banach-Saks property.

Proof. As $X$ is asymptotically uniformly smooth, $X$ cannot contain $\ell_{1}$. Therefore, we only need to notice that $X$ has the $p$-Banach-Saks property, for some $p \in(1, \infty)$, and apply Proposition 3.1. By Theorem 1.2 of $[\mathrm{R}, X$ is asymptotically $p$-uniformly smooth, for some $p \in(1, \infty)$. Therefore, by Proposition 2.2 above, we have that $X$ has the $p$-Banach-Saks property, so we are done.

For each $k \in \mathbb{N}$ and each infinite subset $\mathbb{M} \subset \mathbb{N}$, we define $G_{k}(\mathbb{M})$ as the set of all subsets of $\mathbb{M}$ with $k$ elements. We write $\bar{n}=\left(n_{1}, \ldots, n_{k}\right) \in G_{k}(\mathbb{M})$ always in an increasing order, i.e., $n_{1}<\ldots<n_{k}$. We define a metric $d=d_{k}$ on $G_{k}(\mathbb{M})$ by letting

$$
d(\bar{n}, \bar{m})=\left|\left\{j \mid n_{j} \neq m_{j}\right\}\right|,
$$


for all $\bar{n}=\left(n_{1}, \ldots, n_{k}\right), \bar{m}=\left(m_{1}, \ldots, m_{k}\right) \in G_{k}(\mathbb{M})$.

The following will play an important role in many of the results in this paper. This result was proved in KRa, Theorem 4.2 (see also Theorem 6.1 of [KRa]).

Theorem 3.3. Let $p \in(1, \infty)$, and let $Y$ be a reflexive asymptotically $p$-uniformly smooth Banach space. There exists $K>0$ such that, for all infinite subset $\mathbb{M} \subset \mathbb{N}$, all $k \in \mathbb{N}$, and all bounded map $f: G_{k}(\mathbb{M}) \rightarrow Y$, there exists an infinite subset $\mathbb{M}^{\prime} \subset \mathbb{M}$ such that

$$
\operatorname{diam}\left(f\left(G_{k}\left(\mathbb{M}^{\prime}\right)\right)\right) \leq K L i p(f) k^{1 / p} .
$$

Proof of Theorem 1.3. Let $f: X \rightarrow Y$ be a coarse Lipschitz embedding. Pick $C>0$ so that $\omega_{f}(t) \leq C t+C, \rho_{f}(t) \geq C^{-1} t-C$, for all $t \geq 0$. Assume that $X$ does not have the Banach-Saks property. By $[\mathrm{Be}$, page 373, there exists $\delta>0$ and a sequence $\left(x_{n}\right)_{n=1}^{\infty}$ in $B_{X}$ such that, for all $k \in \mathbb{N}$, and all $n_{1}<\ldots<n_{2 k} \in \mathbb{N}$, we have that

$$
\left\|\frac{1}{2 k} \sum_{j=1}^{k}\left(x_{n_{j}}-x_{n_{k+j}}\right)\right\| \geq \delta .
$$

For each $k \in \mathbb{N}$, define $\varphi_{k}: G_{k}(\mathbb{N}) \rightarrow X$ by setting $\varphi_{k}\left(n_{1}, \ldots, n_{k}\right)=x_{n_{1}}+\ldots+$ $x_{n_{k}}$, for all $\left(n_{1}, \ldots, n_{k}\right) \in G_{k}(\mathbb{N})$. Therefore, $\operatorname{diam}\left(\varphi_{k}\left(G_{k}(\mathbb{M})\right)\right) \geq 2 k \delta$, and we have that $\operatorname{diam}\left(f \circ \varphi_{k}\left(G_{k}(\mathbb{M})\right)\right) \geq 2 k \delta C^{-1}-C$, for all $k \in \mathbb{N}$, and all infinite $\mathbb{M} \subset \mathbb{N}$.

As, $\operatorname{Lip}\left(\varphi_{k}\right) \leq 2$, we have that $\operatorname{Lip}\left(f \circ \varphi_{k}\right) \leq 3 C$. As $Y$ is asymptotically uniformly smooth, there exists $p \in(1, \infty)$ for which $Y$ is asymptotically $p$-uniformly smooth (see $\underline{\mathrm{R}}$, Theorem 1.2). By Theorem 3.3, there exists $K=K(Y)>0$ and $\mathbb{M} \subset \mathbb{N}$ such that $\operatorname{diam}\left(f \circ \varphi_{k}\left(G_{k}(\mathbb{M})\right)\right) \leq 3 K C k^{1 / p}$, for all $k \in \mathbb{N}$. We conclude that

$$
2 k \delta C^{-1}-C \leq 3 K C k^{1 / p},
$$

for all $k \in \mathbb{N}$. As $p>1$, this gives us a contradiction if we let $k \rightarrow \infty$.

The following was asked in GLZ, Problem 2, and it remains open.

Problem 3.4. If a Banach space $X$ coarse Lipschitz embeds into a reflexive asymptotically uniformly smooth Banach space $Y$, does it follow that $X$ has an asymptotically uniformly smooth renorming?

Problem 3.5. Let $N$ be a metric space. We say that a family of metric spaces $\left(M_{k}\right)_{k=1}^{\infty}$ uniformly Lipschitz embeds into $N$ if there exists $C>0$ and Lipschitz embeddings $f_{k}: M_{k} \rightarrow N$ such that $\operatorname{Lip}(f) \cdot \operatorname{Lip}\left(f^{-1}\right)<C$, for all $k \in \mathbb{N}$. Does the family $\left(G_{k}(\mathbb{N}), d\right)_{k=1}^{\infty}$ uniformly Lipschitz embed into any Banach space without an asymptotically uniformly smooth renorming?

As noticed in [GLZ], Problem 6, a positive answer to Problem 3.5 together with Theorem 3.3 would give us a positive answer to Problem 3.4.

It is worth noticing that the Banach-Saks property is not stable under uniform equivalences, hence, it is not stable under coarse Lipschitz isomorphisms either. Indeed, if $\left(p_{n}\right)_{n=1}^{\infty}$ is a sequence in $(1, \infty)$ converging to 1 , then $\left(\oplus_{n} \ell_{p_{n}}\right) \ell_{2}$ is uniformly equivalent to $\left(\oplus_{n} \ell_{p_{n}}\right) \ell_{2} \oplus \ell_{1}$ (see BenLi], page 244). The space $\left(\oplus_{n} \ell_{p_{n}}\right) \ell_{2}$ has the Banach-Saks property, while $\left(\oplus_{n} \ell_{p_{n}}\right) \ell_{2} \oplus \ell_{1}$ does not. 
Let $\mathcal{G}(\mathbb{N})$ denote the set of finite subsets of $\mathbb{N}$. We endow $\mathcal{G}(\mathbb{N})$ with the metric $D$ given by

$$
D(\bar{n}, \bar{m})=|\bar{n} \Delta \bar{m}|
$$

for all $\bar{n}=\left(n_{1}, \ldots, n_{k}\right), \bar{m}=\left(m_{1}, \ldots, m_{l}\right) \in \mathcal{G}(\mathbb{N})$, where $\bar{n} \Delta \bar{m}$ denotes the symmetric difference between the sets $\bar{n}$ and $\bar{m}$.

Proposition 3.6. $\mathcal{G}(\mathbb{N})$ Lipschitz embeds into any Banach space $X$ without the alternating Banach-Saks property. Moreover, for any $\varepsilon>0$, the Lipschitz embedding $f: \mathcal{G}(\mathbb{N}) \rightarrow X$ can be chosen so that $\operatorname{Lip}(f) \cdot \operatorname{Lip}\left(f^{-1}\right)<1+\varepsilon$.

Proof. By Theorem 1 of $[\mathrm{Be}$, page 369, for all $\eta>0$, there exists a bounded sequence $\left(x_{n}\right)_{n=1}^{\infty}$ in $X$ such that, for all $k \in \mathbb{N}$, all $\varepsilon_{1}, \ldots, \varepsilon_{k} \in\{-1,1\}$, and all $n_{1}<\ldots<n_{k}$, we have

$$
1-\eta \leq\left\|\frac{1}{k} \sum_{j=1}^{k} \varepsilon_{j} x_{n_{j}}\right\| \leq 1+\eta .
$$

Define $\varphi: \mathcal{G}(\mathbb{N}) \rightarrow X$ by setting $\varphi\left(n_{1}, \ldots, n_{k}\right)=x_{n_{1}}+\ldots+x_{n_{k}}$, for all $\left(n_{1}, \ldots, n_{k}\right) \in$ $\mathcal{G}(\mathbb{N}) \backslash\{\emptyset\}$, and $\varphi(\emptyset)=0$. Then, we have that

$$
(1-\eta) \cdot D(\bar{n}, \bar{m}) \leq\left\|\varphi_{k}(\bar{n})-\varphi_{k}(\bar{m})\right\| \leq(1+\eta) \cdot D(\bar{n}, \bar{m})
$$

for all $\bar{n}, \bar{m} \in \mathcal{G}(\mathbb{N})$.

Problem 3.7. If $X$ has the Banach-Saks property, does it follow that $\mathcal{G}(\mathbb{N})$ does not Lipschitz embed into $X$ ? In other words, if $X$ is a reflexive Banach space, do we have that $\mathcal{G}(\mathbb{N})$ Lipschitz embed into $X$ if and only if $X$ does not have the Banach-Saks property?

By Corollary 3.2 above, any Banach space with an asymptotically uniformly smooth renorming has the alternating Banach-Saks property. To the best of our knowledge, there is no known example of a Banach space which has the alternating Banach-Saks property but does not admit an asymptotically uniformly smooth renorming. However, using descriptive set theoretical arguments, one can show the existence of such spaces. Recall, $(X, \Omega)$ is called a standard Borel space if $X$ is a set and $\Omega$ is a $\sigma$-algebra on $X$ which is the Borel $\sigma$-algebra associated to a Polish topology on $X$ (i.e., a topology generated by a complete separable metric). A subset $A \subset X$ is called analytic if it is the image of a standard Borel space under a Borel map. We refer to $[\mathrm{Do}]$ and $[\mathrm{Br}$, Section 2, for more details on the descriptive set theory of separable Banach spaces.

Let $C[0,1]$ be the space of continuous real-valued functions on $[0,1]$ endowed with the supremum norm. Let

$$
\mathrm{SB}=\{X \in C[0,1] \mid X \text { is a closed linear subspace }\},
$$

and endow SB with the Effros-Borel structure, i.e., the $\sigma$-algebra generated by

$$
\{X \in \mathrm{SB} \mid X \cap U \neq \emptyset\}, \text { for } U \subset C[0,1] \text { open. }
$$


This makes SB into a standard Borel space and, as $C[0,1]$ contains isometric copies of every separable Banach space, SB can be seen as a coding set for the class of all separable Banach spaces. Therefore, we can talk about Borel and analytic classes of separable Banach spaces.

By $\overline{\mathrm{Br}}$, Theorem 17, the subset $\mathrm{ABS} \subset \mathrm{SB}$ of Banach spaces with the alternating Banach-Saks is not analytic. On the other hand, letting AUS $=\{X \in \mathrm{SB} \mid X$ is asymptotically uniformly smooth\}, we have

$$
X \in \operatorname{AUS} \Leftrightarrow \forall \varepsilon \in \mathbb{Q}_{+} \exists \delta \in \mathbb{Q}_{+} \forall t \in \mathbb{Q}_{+}\left(t<\delta \Rightarrow \bar{\rho}_{X}(t)<\varepsilon t\right) .
$$

As $\{X \in \mathrm{SB} \mid \operatorname{dim}(C[0,1] / X)<\infty\}$ is Borel, it is easy to check that the condition $A(t, \varepsilon) \subset \mathrm{SB}$ given by

$$
X \in A(t, \varepsilon) \Leftrightarrow \bar{\rho}_{X}(t)<\varepsilon t
$$

defines an analytic subset of SB (for similar arguments, we refer to [Do], Chapter 2, Section 2.1). So, AUS must be analytic. Hence, letting AUSable $\subset \mathrm{SB}$ be the subset of Banach spaces with an asymptotically uniformly smooth renorming, we have that

$$
X \in \text { AUSable } \Leftrightarrow \exists Y \in \text { AUS such that } X \cong Y \text {. }
$$

As the isomorphism relation in $\mathrm{SB} \times \mathrm{SB}$ forms an analytic set (see Do, page 11), it follows that AUSable is analytic. This discussion together with Corollary 3.2 gives us the following.

Proposition 3.8. AUSable $\subsetneq A B S$. In particular, there exist separable Banach spaces with the alternating Banach-Saks property which do not admit an asymptotically uniformly smooth renorming.

\section{Asymptotically $p$-Uniformly CONVEX/SMOOTh SPACES.}

In this section, we will use results from $\mathrm{KRa}$ in order to obtain some restrictions on coarse embeddings $X \rightarrow Y$, where the spaces $X$ and $Y$ are assumed to have some asymptotic properties (see Theorem 4.1). We obtain restrictions on the existence of coarse embeddings between the convexified Tsirelson spaces (Theorem 1.5(i)), convexified Schlumprecht spaces (Theorem 1.5(ii)), and some specific hereditarily indecomposable spaces introduced in [D] (Corollary 4.8).

Theorem 4.1. Let $p, q \in(1, \infty)$. Let $X$ be an infinite dimensional Banach space with the p-co-Banach-Saks property and not containing $\ell_{1}$. Let $Y$ be a reflexive asymptotically q-uniformly smooth Banach space. Then, there exists no coarse embedding $f: X \rightarrow Y$ such that

$$
\limsup _{k \rightarrow \infty} \frac{\rho_{f}\left(k^{1 / p}\right)}{k^{1 / q}}=\infty .
$$

Proof. Let $f: X \rightarrow Y$ be a coarse embedding. So, there exists $C>0$, such that $\omega_{f}(t) \leq C t+C$, for all $t>0$. As $X$ does not contain $\ell_{1}$, by Rosenthal's 
$\ell_{1}$-theorem, we can pick a normalized weakly null sequence $\left(x_{n}\right)_{n=1}^{\infty}$ in $X$, with $\inf _{n \neq m}\left\|x_{n}-x_{m}\right\|>0$. For each $k \in \mathbb{N}$, define a map $\varphi_{k}: G_{k}(\mathbb{N}) \rightarrow X$ by letting

$$
\varphi_{k}\left(n_{1}, \ldots, n_{k}\right)=x_{n_{1}}+\ldots+x_{n_{k}}
$$

for all $\left(n_{1}, \ldots, n_{k}\right) \in G_{k}(\mathbb{N})$. So, $\varphi_{k}$ is a bounded map.

If $d\left(\left(n_{1}, \ldots, n_{k}\right),\left(m_{1}, \ldots, m_{k}\right)\right) \leq 1$, then $\left\|\sum_{j=1}^{k} x_{n_{j}}-\sum_{j=1}^{k} x_{m_{j}}\right\| \leq 2$. So, $\operatorname{Lip}\left(f \circ \varphi_{k}\right) \leq 3 C$. By Theorem 3.3, there exists $K=K(Y)>0$ and an infinite subset $\mathbb{M}_{k} \subset \mathbb{N}$ such that

$$
\operatorname{diam}\left(f \circ \varphi_{k}\left(G_{k}\left(\mathbb{M}_{k}\right)\right)\right) \leq 3 K C k^{1 / q} .
$$

Without loss of generality, we may assume that $\mathbb{M}_{k+1} \subset \mathbb{M}_{k}$, for all $k \in \mathbb{N}$. Let $\mathbb{M} \subset \mathbb{N}$ diagonalize the sequence $\left(\mathbb{M}_{k}\right)_{k=1}^{\infty}$, say $\mathbb{M}=\left(n_{j}\right)_{j=1}^{\infty}$. If a sequence $\left(y_{n}\right)_{n=1}^{\infty}$ is weakly null, so is $\left(y_{2 n-1}-y_{2 n}\right)_{n=1}^{\infty}$. Therefore, using the fact that $X$ has the $p$-co-Banach-Saks property to the weakly null sequence $\left(x_{n_{2 j-1}}-x_{n_{2 j}}\right)_{j=1}^{\infty}$, we get that there exists $c>0$ such that, for all $k \in \mathbb{N}$, there exists $m_{1}<\ldots<m_{2 k} \in \mathbb{M}_{k}$, such that

$$
\left\|\sum_{j=1}^{k}\left(x_{m_{2 j-1}}-x_{m_{2 j}}\right)\right\| \geq c k^{1 / p} .
$$

Therefore, we have that $\operatorname{diam}\left(\varphi_{k}\left(G_{k}\left(\mathbb{M}_{k}\right)\right)\right) \geq c k^{1 / p}$, which implies that $\operatorname{diam}(f \circ$ $\left.\varphi_{k}\left(G_{k}\left(\mathbb{M}_{k}\right)\right)\right) \geq \rho_{f}\left(c k^{1 / p}\right)$, for all $k \in \mathbb{N}$. So,

$$
\rho_{f}\left(c k^{1 / p}\right) \leq 3 K C k^{1 / q},
$$

for all $k \in \mathbb{N}$. Therefore, if $\lim \sup _{k \rightarrow \infty} \rho_{f}\left(k^{1 / p}\right) k^{-1 / q}=\infty$, we get a contradiction.

Remark 4.2. Let $X$ be any Banach space containing a sequence $\left(x_{n}\right)_{n=1}^{\infty}$ which is asymptotically $\ell_{1}$, i.e., there exists $L>0$ such that, for all $m \in \mathbb{N}$, there exists $k \in \mathbb{N}$ such that $\left(x_{n_{j}}\right)_{j=1}^{m}$ is $L$-equivalent to $\left(e_{j}\right)_{j=1}^{m}$, for all $k \leq n_{1}<\ldots<n_{m} \in \mathbb{N}$, where $\left(e_{j}\right)_{j=1}^{\infty}$ is the standard $\ell_{1}$-basis. Then, proceeding exactly as above, we can show that there exists no coarse embedding $f: X \rightarrow Y$ such that

$$
\limsup _{k \rightarrow \infty} \frac{\rho_{f}(k)}{k^{1 / q}}=\infty
$$

where $q \in(1, \infty)$ and $Y$ is a reflexive asymptotically $q$-uniformly smooth Banach space.

Let $X$ and $Y$ be Banach spaces. We define $\alpha_{Y}(X)$ as the supremum of all $\alpha>0$ for which there exists a coarse embedding $f: X \rightarrow Y$ and $L>0$ such that

$$
L^{-1}\|x-y\|^{\alpha}-L \leq\|f(x)-f(y)\|,
$$

for all $x, y \in X$. We call $\alpha_{Y}(X)$ the compression exponent of $X$ in $Y$, or the $Y$ compression of $X$. If, for all $\alpha>0$, no such $f$ and $L$ exist, we set $\alpha_{Y}(X)=0$. As 
$\omega_{f}$ is always bounded by an affine map (as $X$ is a Banach space), it follows that $\alpha_{Y}(X) \in[0,1]$. Also, $\alpha_{Y}(X)=0$ if $X$ does not coarsely embed into $Y$.

The quantity $\alpha_{Y}(X)$ was first introduced by E. Guentner and J. Kaminker in GuKa. For a detailed study of $\alpha_{\ell_{q}}\left(\ell_{p}\right), \alpha_{L_{q}}\left(\ell_{p}\right), \alpha_{\ell_{q}}\left(L_{p}\right)$, and $\alpha_{L_{q}}\left(L_{p}\right)$, where $p, q \in(0, \infty)$, we refer to $\mathrm{B}$.

Using this terminology, let us reinterpret Theorem 4.1

Theorem 4.3. Let $1<p<q$. Let $Y$ be a reflexive asymptotically q-uniformly smooth Banach space. The following holds.

(i) If $X$ contains a sequence which is asymptotically $\ell_{1}$, then $\alpha_{Y}(X) \leq 1 / q$.

(ii) If $X$ is an infinite dimensional Banach space with the p-co-Banach-Saks property and not containing $\ell_{1}$, then $\alpha_{Y}(X) \leq p / q$.

In particular, $X$ does not coarse Lipschitz embed into $Y$.

Proof. (ii) Let $L>0$ and $f: X \rightarrow Y$ be a coarse embedding such that $\rho_{f}(t) \geq$ $L^{-1} t^{\alpha}-L$, for all $t>0$. By Theorem 4.1, we must have

$$
\limsup _{k \rightarrow \infty} k^{\alpha / p-1 / q} L^{-1}-L k^{-1 / q}<\infty .
$$

Therefore, $\alpha / p-1 / q \leq 0$, and the result follows.

(i) This follows from Remark 4.2 and the same reasoning as item (ii) above.

Notice that $Y$ being reflexive in Theorem 4.3 cannot be removed. Indeed, $c_{0}$ contains a Lipschitz copy of any separable metric space (see [A]), and it is also asymptotically $q$-uniformly smooth, for any $q \in(1, \infty)$.

Corollary 4.4. Let $1<p<q$. Let $X$ be asymptotically $p$-uniformly convex, and $Y$ be reflexive and asymptotically q-uniformly smooth. Then $\alpha_{Y}(X) \leq p / q$.

Asking the Banach space $X$ to have the $p$-co-Banach-Saks property in Theorem 4.3 is actually too much, and we can weaken this condition by only requiring $X$ to have the almost $p$-co-Banach-Saks property. Precisely, we have the following.

Theorem 4.5. Let $1<p<q$. Let $X$ be an infinite dimensional Banach space with the almost $p$-co-Banach-Saks property. Let $Y$ be a reflexive asymptotically $q$ uniformly smooth Banach space. Then $\alpha_{Y}(X) \leq p / q$. In particular, $X$ does not coarse Lipschitz embed into $Y$.

Proof. Let $f: X \rightarrow Y$ be a coarse embedding and pick $C>0$ such that $\omega_{f}(t) \leq$ $C t+C$, for all $t \geq 0$. If $X$ contains $\ell_{1}$, the result follows from Theorem 4.3(i). If $X$ does not contain $\ell_{1}$, we can pick a normalized weakly null sequence $\left(x_{n}\right)_{n=1}^{\infty}$ in $X$, with $\inf _{n \neq m}\left\|x_{n}-x_{m}\right\|>0$. By taking a subsequence of $\left(x_{n}\right)_{n=1}^{\infty}$ if necessary, pick $\left(\theta_{k}\right)_{k=1}^{\infty}$ as in the definition of the almost $p$-co-Banach-Saks property. Define $\varphi_{k}: G_{k}(\mathbb{N}) \rightarrow X$ by letting $\varphi_{k}\left(n_{1}, \ldots, n_{k}\right)=x_{n_{1}}+\ldots+x_{n_{k}}$, for all $\left(n_{1}, \ldots, n_{k}\right) \in$ $G_{k}(\mathbb{N})$.

Following the proof of Theorem 4.1, we get that

$$
\rho_{f}\left(k^{1 / p} \theta_{k}^{-1}\right) \leq 3 K C k^{1 / q},
$$

for all $k \in \mathbb{N}$. Let $L>0$ and $\alpha>0$ be such that $\rho_{f}(t) \geq L^{-1} t^{\alpha}-L$, for all $t>0$. Then, 


$$
k^{\alpha / p-1 / q} \theta_{k}^{-\alpha} L^{-1} \leq 4 K C,
$$

for big enough $k \in \mathbb{N}$. As $\lim _{k \rightarrow \infty} k^{\beta} \theta_{k}^{-\alpha}=\infty$, for all $\beta>0$, we must have that $\alpha / p-1 / q \leq 0$.

Remark 4.6. Let $\left(x_{n}\right)_{n=1}^{\infty}$ be a bounded sequence in a Banach space $X$ with the following property: there exists a sequence of positive reals $\left(\theta_{j}\right)_{j=1}^{\infty}$ in $[1, \infty)$ such that $\lim _{j \rightarrow \infty} j^{\alpha} \theta_{j}^{-1}=\infty$, for all $\alpha>0$, and

$$
k \theta_{k}^{-1} \leq\left\| \pm x_{n_{1}}+\ldots+ \pm x_{n_{k}}\right\|
$$

for all $n_{1}<\ldots<n_{k} \in \mathbb{N}$. The proof of Theorem 4.5 gives us that $\alpha_{Y}(X) \leq 1 / q$, for any reflexive asymptotically $q$-uniformly smooth Banach space $Y$, with $q>1$.

Let $q>1$, and let $\left(E_{n}\right)_{n=1}^{\infty}$ be a sequence of finite dimensional Banach spaces. Let $\mathcal{E}$ be a 1 -unconditional basic sequence. Notice that, if $\mathcal{E}$ generates a reflexive asymptotically $q$-uniformly smooth Banach space, then $\left(\oplus_{n} E_{n}\right)_{\mathcal{E}}$ is also reflexive and asymptotically $q$-uniformly smooth. Hence, Theorem 4.3 and Theorem 4.5 gives us the following corollary.

Corollary 4.7. Let $1<p<q$, and let $\left(E_{n}\right)_{n=1}^{\infty}$ be a sequence of finite dimensional Banach spaces. Let $\mathcal{E}$ be a 1-unconditional basic sequence generating a reflexive asymptotically q-uniformly smooth Banach space. The following holds.

(i) If $X$ contains a sequence with Property (囝), then $\alpha_{\left(\oplus_{n} E_{n}\right)_{\mathcal{E}}}(X) \leq 1 / q$.

(ii) If $X$ is an infinite dimensional Banach space with the almost $p$-co-BanachSaks property, then $\alpha_{\left(\oplus_{n} E_{n}\right)_{\mathcal{E}}}(X) \leq p / q$.

In particular, $X$ does not coarse Lipschitz embed into $\left(\oplus_{n} E_{n}\right)_{\mathcal{E}}$.

Proof of Theorem 1.5. (i) As noticed in Subsection 2.6. $T^{p}$ has the $p$-co-BanachSaks property, and is asymptotically $p$-uniformly smooth, for all $p \in(1, \infty)$. Therefore, as $T^{p}$ is reflexive (see OScZs, Proposition 5.3(b)), for all $p \in[1, \infty$ ), the result follows from Theorem 4.3 (or Corollary 4.7).

(ii) For any $p \in(1, \infty), S^{p}$ has the almost $p$-co-Banach-Saks property and is asymptotically $p$-uniformly smooth. By Theorem 8 and Proposition 2(2) of CKKuM, $S^{p}$ is reflexive, for all $p \in[1, \infty)$. So, the result follows from Corollary 4.7.

A Banach space $X$ is called hereditarily indecomposable if none of its subspaces can be decomposed as a sum of two infinite dimensional Banach spaces. In Chapter 5 of $[\mathrm{D}$, for each $p \in(1, \infty)$, Dew constructed a hereditarily indecomposable space $\mathfrak{X}_{p}$ with a basis $\left(e_{n}\right)_{n=1}^{\infty}$ satisfying the following properties: (i) $\mathfrak{X}_{p}$ is reflexive, (ii) the base $\left(e_{n}\right)_{n=1}^{\infty}$ satisfies an upper $\ell_{p}$-estimate with constant 1 , and (iii) if $\left(x_{n}\right)_{n=1}^{\infty}$ is a block sequence of $\left(e_{n}\right)_{n=1}^{\infty}$, then, for all $n \in \mathbb{N}$,

$$
\left\|\sum_{j=1}^{n} x_{j}\right\| \geq f(n)^{-1 / p}\left(\sum_{j=1}^{n}\left\|x_{j}\right\|^{p}\right)^{1 / p},
$$

where $f: \mathbb{N} \rightarrow[0, \infty)$ is a function such that, among other properties, $\lim _{n \rightarrow \infty} n^{\alpha} f(n)^{-1}$ $=\infty$, for all $\alpha>0$. In particular, $\mathfrak{X}_{p}$ has the almost $p$-co-Banach-Saks property, 
and it is asymptotically $p$-uniformly smooth. This, together with Theorem 4.5 gives us the following.

Corollary 4.8. Let $1<p<q$. Then $\alpha_{\mathfrak{X}^{q}}\left(\mathfrak{X}^{p}\right) \leq p / q$. In particular, $\mathfrak{X}_{p}$ does not coarse Lipschitz embeds into $\mathfrak{X}_{q}$.

Problem 4.9. Let $1 \leq p<q$. Does $\alpha_{T^{q}}\left(T^{p}\right)=\alpha_{S^{q}}\left(S^{p}\right)=p / q$ ? If $p>1$, does $\alpha_{\mathfrak{X}^{q}}\left(\mathfrak{X}^{p}\right)=p / q$ hold?

Remark 4.10. It is worth noticing that, if $p>\max \{q, 2\}$, then $\alpha_{T^{q}}\left(T^{p}\right)=0$. Indeed, for all $r \geq 2, T^{r}$ has cotype $r+\varepsilon$ for all $\varepsilon>0$ (see DiJT, page 305). On the other hand, if $r<2$, then $T^{r}$ has cotype 2. This follows from the fact that, for any $\varepsilon>0$, $T^{r}$ has an equivalent norm satisfying a lower $\ell_{(r+\varepsilon)}$-estimate (we explain this in the proof of Corollary [1.6 below), then, by Theorem 1.f.7 and Proposition 1.f.3(i) of LiT], $T^{r}$ has cotype 2. Similarly, by Theorem 1.f.7 and Proposition 1.f.3(ii), $T^{r}$ has non trivial type, for all $r \in(1, \infty)$. By Theorem 1.11 of [MeN], if a Banach space $X$ coarsely embeds into a Banach space $Y$ with non trivial type, then

$$
\inf \{q \in[2, \infty) \mid X \text { has cotype } q\} \leq \inf \{q \in[2, \infty) \mid Y \text { has cotype } q\}
$$

Therefore, we conclude that $T^{p}$ does not coarsely embed into $T^{q}$, if $p>\max \{q, 2\}$. So, $\alpha_{T^{q}}\left(T^{p}\right)=0$.

Problem 4.11. Let $1 \leq q<p \leq 2$. What can we say about $\alpha_{T^{q}}\left(T^{p}\right)$ ?

We finish this section with an application of Theorem 4.3, Theorem 4.5. and Theorem 3.4 of $\mathrm{AlB}$. By looking at the proof of Theorem 3.4 of $\mathrm{AlB}$, one can easily see that the authors proved a stronger result than the one stated in their paper. Precisely, the authors proved the following.

Theorem 4.12. Let $0<p<q$. There exist maps $\left(\psi_{j}: \mathbb{R} \rightarrow \mathbb{R}\right)_{j=1}^{\infty}$ such that, for all $x, y \in \mathbb{R}$,

$$
A_{p, q}|x-y|^{p} \leq \max \left\{\left|\psi_{j}(x)-\psi_{j}(y)\right|^{q} \mid j \in \mathbb{N}\right\}
$$

and

$$
\sum_{j \in \mathbb{N}}\left|\psi_{j}(x)-\psi_{j}(y)\right|^{q} \leq B_{p, q}|x-y|^{p},
$$

where $A_{p, q}, B_{p, q}$ are positive constants.

Proposition 4.13. Let $1 \leq p<q$. There exists a map $f: T^{p} \rightarrow\left(\oplus T^{q}\right)_{T^{q}}$ which is simultaneously a coarse and a uniform embedding such that $\rho_{f}(t) \geq C t^{p / q}$, for some $C>0$. In particular, $\alpha_{\left(\oplus T^{q}\right)_{T^{q}}}\left(T^{p}\right)=p / q$.

Proof. Let $\left(\psi_{j}\right)_{j=1}^{\infty}, A_{p, q}$, and $B_{p, q}$ be given by Theorem 4.12. Define $f: T^{p} \rightarrow$ $\left(\oplus T_{q}\right)_{T^{q}}$ by letting

$$
f(x)=\left(\left(\psi_{j}\left(x_{n}\right)-\psi_{j}(0)\right)_{j=1}^{\infty}\right)_{n=1}^{\infty}
$$

for all $x=\left(x_{n}\right)_{n=1}^{\infty} \in T^{p}$. One can easily check that $f$ satisfies 


$$
A_{p, q}^{1 / q}\|x-y\|^{p / q} \leq\|f(x)-f(y)\| \leq B_{p, q}^{1 / q}\|x-y\|^{p / q},
$$

for all $x, y \in T^{p}$.

As $T^{q}$ is $q$-convex, it is easy to see that $\left(\oplus T^{q}\right)_{T^{q}}$ is asymptotic $q$-uniformly smooth. Hence, as $\left(\oplus T^{q}\right)_{T^{q}}$ is reflexive, we conclude that $\alpha_{\left(\oplus T^{q}\right)_{T^{q}}}\left(T^{p}\right)=p / q$.

Corollary 4.14. T strongly embeds into a super-reflexive Banach space.

Proof. It is easy to check that $\left(\oplus T^{2}\right)_{T^{2}}$ is super-reflexive. Indeed, super-reflexivity is equivalent to a uniformly convex renorming. Hence, if $\mathcal{E}$ is a 1-unconditional basis generating a super-reflexive space, and $X$ is a super-reflexive space, then so is $(\oplus X)_{\mathcal{E}}$ (see [LiT], page 100).

Similarly as above, we get the following proposition.

Proposition 4.15. Let $1 \leq p<q$. There exists a map $f: S^{p} \rightarrow\left(\oplus S^{q}\right)_{S^{q}}$ which is simultaneously a coarse and a uniform embedding such that $\rho_{f}(t) \geq C t^{p / q}$, for some $C>0$. In particular, $\alpha_{\left(\oplus S^{q}\right)_{S q}}\left(S^{p}\right)=p / q$.

\section{CoArse Lipschitz Embeddings into sums.}

In this last section, we will be specially interested in the nonlinear geometry of the Tsirelson space and its convexifications. In order to obtain Theorem 1.6, we will prove a technical result on the coarse Lipschitz non embeddability of certain Banach spaces into the direct sum of Banach spaces with certain $p$-properties (Theorem 5.6). The main goal of this section is to characterize the Banach spaces which are coarsely (resp. uniformly) equivalent to $T^{p_{1}} \oplus \ldots \oplus T^{p_{n}}$, for $p_{1}, \ldots, p_{n} \in(1, \ldots, \infty)$, and $2 \notin\left\{p_{1}, \ldots, p_{n}\right\}$.

Given $x, y \in X$, and $\delta>0$ the approximate midpoint between $x$ and $y$ with error $\delta$ is given by

$$
\operatorname{Mid}(x, y, \delta)=\left\{z \in X \mid \max \{\|x-z\|,\|y-z\|\} \leq 2^{-1}(1+\delta)\|x-y\|\right\} .
$$

The following lemma is an asymptotic version of Lemma 1.6(i) of JoLiS and Lemma 3.2 of KRa.

Lemma 5.1. Let $X$ be an asymptotically p-uniformly smooth Banach space, for some $p \in(1, \infty)$. There exists $c>0$ such that, for all $x, y \in X$, all $\delta>0$, and all weakly null sequence $\left(x_{n}\right)_{n=1}^{\infty}$ in $B_{X}$, there exists $n_{0} \in \mathbb{N}$ such that, for all $n>n_{0}$, we have

$$
u+\delta^{1 / p}\|v\| x_{n} \in \operatorname{Mid}(x, y, c \delta)
$$

where $u=\frac{1}{2}(x+y)$, and $v=\frac{1}{2}(x-y)$.

Proof. By Proposition 1.3 of [DimGoJ], there exists $c>0$ such that, for all weakly null sequence $\left(x_{n}\right)_{n=1}^{\infty}$ in $B_{X}$, we have

$$
\underset{n}{\limsup }\left\|x+x_{n}\right\|^{p} \leq\|x\|^{p}+c \cdot \limsup _{n}\left\|x_{n}\right\|^{p} .
$$


Fix such sequence. As $\left\|x-\left(u+\delta^{1 / p}\|v\| x_{n}\right)\right\|=\left\|v-\delta^{1 / p}\right\| v\left\|x_{n}\right\|$, we get

$$
\underset{n}{\limsup }\left\|x-\left(u+\delta^{1 / p}\|v\| x_{n}\right)\right\|^{p} \leq(1+c \delta)\|v\|^{p} .
$$

Therefore, as $(1+c \delta)^{1 / p}<1+c \delta$, there exists $n_{0} \in \mathbb{N}$ such that $\| x-(u+$ $\left.\delta^{1 / p}\|v\| x_{n}\right)\|\leq(1+c \delta)\| v \|$, for all $n>n_{0}$. Similarly, we can assume that $\| y-$ $\left(u+\delta^{1 / p}\|v\| x_{n}\right)\|\leq(1+c \delta)\| v \|$, for all $n>n_{0}$.

The following lemma is a simple modification of Lemma 3.3 of [KRa], or Lemma 1.6(ii) of [JoLiS], so we omit its proof.

Lemma 5.2. Suppose $1 \leq p<\infty$, and let $X$ be Banach space with a 1-unconditional basis $\left(e_{n}\right)_{n=1}^{\infty}$ satisfying a lower $\ell_{p}$-estimate with constant 1 . For all $x, y \in X$, and all $\delta>0$, there exists a compact subset $K \subset X$, such that

$$
\operatorname{Mid}(x, y, \delta) \subset K+2 \delta^{1 / p}\|v\| B_{X},
$$

where $u=\frac{1}{2}(x+y)$, and $v=\frac{1}{2}(x-y)$.

For each $s>0$, let

$$
\operatorname{Lip}_{s}(f)=\sup _{t \geq s} \frac{\omega_{f}(t)}{t} \text { and } \operatorname{Lip}_{\infty}(f)=\inf _{s>0} \operatorname{Lip}_{s}(f) .
$$

We will need the following proposition, which can be found in KRa as Proposition 3.1 .

Proposition 5.3. Let $X$ be a Banach space and $M$ be a metric space. Let $f: X \rightarrow$ $M$ be a coarse map with $\operatorname{Lip}_{\infty}(f)>0$. Then, for all $\varepsilon, t>0$, and all $\delta \in(0,1)$, there exists $x, y \in X$ with $\|x-y\|>t$ such that

$$
f(\operatorname{Mid}(x, y, \delta)) \subset \operatorname{Mid}(f(x), f(y),(1+\varepsilon) \delta) .
$$

The following lemma will play the same role in our settings as Proposition 3.5 did in KRa.

Lemma 5.4. Let $1 \leq q<p$. Let $X$ be an asymptotically $p$-uniformly smooth Banach space, and $Y$ be a Banach space with a 1-unconditional basis satisfying a lower $\ell_{q}$-estimate with constant 1 . Let $f: X \rightarrow Y$ be a coarse map. Then, for any $t>0$, and any $\delta \in(0,1)$, there exists $x \in X, \tau>t$, and a compact subset $K \subset Y$ such that, for any weakly null sequence $\left(x_{n}\right)_{n=1}^{\infty}$ in $B_{X}$, there exists $n_{0} \in \mathbb{N}$ such that

$$
f\left(x+\tau x_{n}\right) \in K+\delta \tau B_{Y}, \quad \text { for all } n>n_{0} .
$$

Proof. If $\operatorname{Lip}_{\infty}(f)=0$, then there exists $\tau>t$ such that $\operatorname{Lip}_{\tau}(f)<\delta$. Hence, $\omega_{f}(\tau)<\delta \tau$, and the result follows by letting $x=0$ and $K=\{f(0)\}$. Indeed, if $z \in B_{X}$, we have

$$
\|f(\tau z)-f(0)\| \leq \omega_{f}(\|\tau z\|) \leq \omega_{f}(\tau) \leq \delta \tau
$$


Assume $\operatorname{Lip}_{\infty}(f)>0$. In particular, $C=\operatorname{Lip}_{s}(f)>0$, for some $s>0$. Let $c>0$ be given by Lemma 5.1 applied to $X$ and $p$. As $q<p$, we can pick $\nu \in(0,1)$ such that $2 C(2 c)^{1 / q} \nu^{1 / q-1 / p}<\delta$. By Proposition 5.3, there exists $u, v \in X$ such that $\|u-v\|>\max \left\{s, 2 t \nu^{-1 / p}\right\}$ and

$$
f(\operatorname{Mid}(u, v, c \nu)) \subset \operatorname{Mid}(f(u), f(v), 2 c \nu) .
$$

Let $x=\frac{1}{2}(u+v)$, and $\tau=\nu^{1 / p}\left\|\frac{1}{2}(u-v)\right\|$ (so $\left.\tau>t\right)$. Fix a weakly null sequence $\left(x_{n}\right)_{n=1}^{\infty}$ in $B_{X}$. Then, by Lemma [5.1, there exists $n_{0} \in \mathbb{N}$ such that $x+\tau x_{n} \in \operatorname{Mid}(u, v, c \nu)$, for all $n>n_{0}$. So,

$$
f\left(x+\tau x_{n}\right) \subset f(\operatorname{Mid}(u, v, c \nu)) \subset \operatorname{Mid}(f(u), f(v), 2 c \nu),
$$

for all $n>n_{0}$. Let $K \subset Y$ be given by Lemma 5.2 applied to $Y, f(u), f(v) \in Y$, and $2 c \nu$. So,

$$
\operatorname{Mid}(f(u), f(v), 2 c \nu) \subset K+2(2 c)^{1 / q} \nu^{1 / q} \frac{\|f(u)-f(v)\|}{2} B_{Y} .
$$

As $\operatorname{Lip}_{s}(f)=C$, and as $\|u-v\|>s$, we have $\|f(u)-f(v)\| \leq C\|u-v\|=2 C \tau \nu^{-1 / p}$. Hence,

$$
2(2 c)^{1 / q} \nu^{1 / q} \frac{\|f(u)-f(v)\|}{2} \leq 2 C(2 c)^{1 / q} \nu^{1 / q-1 / p} \tau<\delta \tau,
$$

and we are done.

Remark 5.5. Lemma 5.4 remains valid if we only assume that $X$ has an equivalent norm with which $X$ becomes asymptotically $p$-uniformly smooth. Indeed, let $M \geq 1$ be such that $B_{(X,\|\cdot\|)} \subset M \cdot B_{(X,\|\cdot\|)}$. Fix $t>0$, and $\delta \in(0,1)$. Applying Lemma 5.4 to $(X,\|\cdot\| \|)$ with $t^{\prime}=M . t$ and $\delta^{\prime}=\delta / M$, we obtain $x \in X, \tau^{\prime}>t^{\prime}$, and a compact set $K \subset Y$. The result now follows by letting $\tau=\tau^{\prime} / M$.

Theorem 5.6. Let $1 \leq q_{1}<p<q_{2}$. Assume that

(i) $X$ is an asymptotically $p$-uniformly smooth Banach space with the p-co-BanachSaks property, and it does not contain $\ell_{1}$,

(ii) $Y_{1}$ is a Banach space with a 1-unconditional basis satisfying a lower $\ell_{q_{1}}$ estimate with constant 1 , and

(iii) $Y_{2}$ is a reflexive asymptotically $q_{2}$-uniformly smooth Banach space.

Then $X$ does not coarse Lipschitz embed into $Y_{1} \oplus Y_{2}$.

Proof. Let $Y_{1} \oplus_{1} Y_{2}$ denote the space $Y_{1} \oplus Y_{2}$ endowed with the norm $\left\|\left(y_{1}, y_{2}\right)\right\|=$ $\left\|y_{1}\right\|+\left\|y_{2}\right\|$, for all $\left(y_{1}, y_{2}\right) \in Y_{1} \oplus Y_{2}$. Assume $f=\left(f_{1}, f_{2}\right): X \rightarrow Y_{1} \oplus_{1} Y_{2}$ is a coarse Lipschitz embedding. As $f$ is a coarse Lipschitz embedding, there exists $C>0$ such that $\rho_{f}(t) \geq C^{-1} t-C$, and $\omega_{f_{2}}(t) \leq C t+C$, for all $t>0$.

Fix $k \in \mathbb{N}$, and $\delta \in(0,1)$. Then, by Lemma [5.4, there exists $\tau>k, x \in X$, and a compact subset $K \subset Y_{1}$, such that, for any weakly null sequence $\left(y_{n}\right)_{n=1}^{\infty}$ in $B_{X}$, there exists $n_{0} \in \mathbb{N}$, such that

$$
f_{1}\left(x+\tau y_{n}\right) \in K+\delta \tau B_{Y_{1}},
$$


for all $n>n_{0}$.

As $X$ does not contain $\ell_{1}$, by Rosenthal's $\ell_{1}$-theorem, we can pick a normalized weakly null sequence $\left(x_{n}\right)_{n=1}^{\infty}$ in $X$, with $\inf _{n \neq m}\left\|x_{n}-x_{m}\right\|>0$. As $X$ has the $p$-Banach-Saks property (Proposition 2.2), there exists $c>0$ (independent of $k$ ) such that, by going to a subsequence if necessary, we have

$$
\left\|x_{n_{1}}+\ldots+x_{n_{k}}\right\| \leq c k^{1 / p},
$$

for all $n_{1}<\ldots<n_{k} \in \mathbb{N}$. Define a map $\varphi_{k, \delta}: G_{k}(\mathbb{N}) \rightarrow X$ by letting

$$
\varphi_{k, \delta}\left(n_{1}, \ldots, n_{k}\right)=x+\frac{\tau}{c} k^{-1 / p}\left(x_{n_{1}}+\ldots+x_{n_{k}}\right),
$$

for all $\left(n_{1}, \ldots, n_{k}\right) \in G_{k}(\mathbb{N})$.

As $d\left(\left(n_{1}, \ldots, n_{k}\right),\left(m_{1}, \ldots, m_{k}\right)\right) \leq 1$ implies $\left\|\sum_{j=1}^{k} x_{n_{j}}-\sum_{j=1}^{k} x_{m_{j}}\right\| \leq 2$, we have that $\operatorname{Lip}\left(f_{2} \circ \varphi_{k, \delta}\right) \leq 2 \tau C k^{-1 / p} c^{-1}+C$. Therefore, by Theorem 3.3, there exists $\mathbb{M}_{k, \delta} \subset \mathbb{N}$ such that

$$
\operatorname{diam}\left(f_{2} \circ \varphi_{k, \delta}\left(G_{k}\left(\mathbb{M}_{k, \delta}\right)\right)\right) \leq 2 K \tau C k^{1 / q_{2}-1 / p} c^{-1}+K C k^{1 / q_{2}},
$$

for some $K>0$ independent of $k$ and $\delta$.

Notice that, if $\left(n_{1}^{j}, \ldots, n_{k}^{j}\right)_{j=1}^{\infty}$ is a sequence in $G_{k}\left(\mathbb{M}_{k, \delta}\right)$, with $n_{k}^{j}<n_{1}^{j+1}$, for all $j \in \mathbb{N}$, then $\left(x_{n_{1}^{j}}+\ldots+x_{n_{k}^{j}}\right)_{j=1}^{\infty}$ is a weakly null sequence in $c k^{1 / p} \cdot B_{X}$. Therefore,

$$
f_{1} \circ \varphi_{k, \delta}\left(n_{1}^{j}, \ldots, n_{k}^{j}\right) \in K+\delta \tau B_{Y_{1}},
$$

for large enough $j$. This argument and standard Ramsey theory, gives us that, by passing to a subsequence of $\mathbb{M}_{k, \delta}$, we can assume that, for all $\left(n_{1}, \ldots, n_{k}\right) \in$ $G_{k}\left(\mathbb{M}_{k, \delta}\right)$,

$$
f_{1} \circ \varphi_{k, \delta}\left(n_{1}, \ldots, n_{k}\right) \in K+\delta \tau B_{Y_{1}} .
$$

Therefore, as $K$ is compact, by passing to a further subsequence, we can assume that $\operatorname{diam}\left(f_{1} \circ \varphi_{k, \delta}\left(G_{k}\left(\mathbb{M}_{k, \delta}\right)\right)\right) \leq 3 \delta \tau$ (see Lemma 4.1 of [KRa]).

We have shown that, for all $k \in \mathbb{N}$, and all $\delta \in(0,1)$, there exists a subsequence $\mathbb{M}_{k, \delta} \subset \mathbb{N}$ such that

$$
\operatorname{diam}\left(f \circ \varphi_{k, \delta}\left(G_{k}\left(\mathbb{M}_{k, \delta}\right)\right)\right) \leq 2 K \tau C k^{1 / q_{2}-1 / p} c^{-1}+K C k^{1 / q_{2}}+3 \delta \tau .
$$

We may assume that $\mathbb{M}_{k+1, \delta} \subset \mathbb{M}_{k, \delta}$, for all $k \in \mathbb{N}$, and all $\delta \in(0,1)$. For each $\delta \in(0,1)$, let $\mathbb{M}_{\delta} \subset \mathbb{N}$ diagonalize the sequence $\left(\mathbb{M}_{k, \delta}\right)_{k=1}^{\infty}$.

As $X$ has the $p$-co-Banach-Saks property, arguing similarly as in the proof of Theorem 4.1 we get that there exists $d>0$ (independent of $k$ ) such that, for all $k \in \mathbb{N}$, there exists $n_{1}<\ldots<n_{2 k} \in \mathbb{M}_{k, \delta}$, such that 


$$
\left\|\sum_{j=1}^{k}\left(x_{n_{2 j-1}}-x_{n_{2 j}}\right)\right\| \geq d k^{1 / p} .
$$

Therefore, $\operatorname{diam}\left(\varphi_{k, \delta}\left(G_{k}\left(\mathbb{M}_{\delta}\right)\right)\right) \geq \tau d / c$, which implies that

$$
\operatorname{diam}\left(f \circ \varphi_{k, \delta}\left(G_{k}\left(\mathbb{M}_{\delta}\right)\right)\right) \geq \tau d(c C)^{-1}-C,
$$

for all $k \in \mathbb{N}$, and all $\delta \in(0,1)$. So, Equation (5.1) and Equation (5.2) give us that

$$
\tau d(c C)^{-1}-C \leq 2 K \tau C k^{1 / q_{2}-1 / p} c^{-1}+K C k^{1 / q_{2}}+3 \delta \tau .
$$

for all $k \in \mathbb{N}$, and all $\delta \in(0,1)$. As $\tau>k$, this gives us that

$$
d(c C)^{-1}-C k^{-1} \leq 2 K C k^{1 / q_{2}-1 / p} c^{-1}+K C k^{1 / q_{2}-1}+3 \delta
$$

for all $k \in \mathbb{N}$, and all $\delta \in(0,1)$. As $q_{2}>p>1$, by letting $k \rightarrow \infty$ and $\delta \rightarrow 0$, we get a contradiction.

If $T=\left(T_{1}, T_{2}\right): X \rightarrow Y_{1} \oplus Y_{2}$ is a linear isomorphic embedding, then either $T_{1}: X \rightarrow Y_{1}$ or $T_{2}: X \rightarrow Y_{2}$ is not strictly singular, i.e., $T_{i}: X_{0} \rightarrow Y_{i}$ is a linear isomorphic embedding, for some infinite dimensional subspace $X_{0} \subset X$, and some $i \in\{1,2\}$. Is there an analog of this result for coarse Lipschitz embeddings? Precisely, we ask the following.

Problem 5.7. Let $X, Y_{1}$ and $Y_{2}$ be Banach spaces and consider a coarse Lipschitz embedding $f=\left(f_{1}, f_{2}\right): X \rightarrow Y_{1} \oplus Y_{2}$. Is there an infinite dimensional subspace $X_{0} \subset X$ such that either $f_{1}: X_{0} \rightarrow Y_{1}$ or $f_{2}: X_{0} \rightarrow Y_{2}$ is a coarse Lipschitz embedding?

We can now prove Theorem[1.6, which will be essential in the proof of Theorem 1.7.

Proof of Theorem 1.6. Say $m \in\{1, \ldots, n-1\}$ is such that $p \in\left(p_{m}, p_{m+1}\right)$ (the other cases have analogous proofs). Then $\left(T^{p_{m+1}} \oplus \ldots \oplus T^{p_{n}}\right)_{\ell_{\infty}}$ is reflexive (see OScZs, Proposition 5.3(b)). Also, it is easy to see that $\left(T^{p_{m+1}} \oplus \ldots \oplus T^{p_{n}}\right)_{\ell_{\infty}}$ is asymptotically $p_{m+1}$-uniformly smooth. By Theorem 5.6, it is enough to prove the following claim.

Claim: Fix $\varepsilon>0$ such that $p_{m}+\varepsilon<p .\left(T^{p_{1}} \oplus \ldots \oplus T^{p_{m}}\right)_{\ell_{p_{m}}}$ can be renormed so that it has a 1 -unconditional basis satisfying a lower $\ell_{\left(p_{m}+\varepsilon\right)}$-estimate with constant 1.

For each $k \in \mathbb{N}$ and $p \in[1, \infty)$, denote by $P_{k}=P_{k}^{p}: T^{p} \rightarrow T^{p}$ the projection on the first $k$ coordinates, and let $Q_{k}=\mathrm{Id}-P_{k}$. By Proposition 5.6 of JoLiS, there exists $M \in[1, \infty)$ and $N \in \mathbb{N}$ such that $Q_{N}\left(T^{p_{j}}\right)$ has an equivalent norm with $\left(p_{j}+\varepsilon\right)$-concavity constant $M$, for all $j \in\{1, \ldots, m\}$ (precisely, the modified Tsirelson norm has this property, see [CSh] for definition).

As the shift operator on the basis of $T^{p}$ is an isomorphism onto $Q_{1}\left(T^{p}\right)$, we have that $T^{p} \cong Q_{k}\left(T^{p}\right)$, for all $k \in \mathbb{N}$, and all $p \in[1, \infty)$. Therefore, it follows that 
$\left(T^{p_{1}} \oplus \ldots \oplus T^{p_{m}}\right)_{\ell_{p_{m}}}$ has an equivalent norm with $\left(p_{m}+\varepsilon\right)$-concavity constant $M$. By Proposition 1.d.8 of [LiT], we can assume that $M=1$. As a $q$-concave basis with constant 1 satisfies a lower $\ell_{q}$-estimate with constant 1 , we are done.

Before given the proof of Theorem 1.7 we need a lemma. For that, we must introduce some natation. Let $p \in(1, \infty)$. A Banach space $X$ is said to be as. $\mathcal{L}_{p}$ if there exists $\lambda>0$ so that for every $n \in \mathbb{N}$ there is a finite codimensional subspace $Y \subset X$ so that every $n$-dimensional subspace of $Y$ is contained in a subspace of $X$ which is $\lambda$-isomorphic to $L_{p}(\mu)$, for some $\mu$. As noticed in JoLiS, Proposition 2.4.a, an as. $\mathcal{L}_{p}$ space is super-reflexive. Also, the $p$-convexifications $T^{p}$ are as. $\mathcal{L}_{p}$ (see [JoLiS], page 440).

The following lemma, although not explicitily written, is contained in the proof of Proposition 2.7 of [JoLiS]. For the convenience of the reader, we provide its proof here.

Lemma 5.8. Say $1<p_{1}<\ldots<p_{n}<\infty$ and $X=X^{p_{1}} \oplus \ldots \oplus X^{p_{n}}$, where $X^{p_{j}}$ is as. $\mathcal{L}_{p_{j}}$, for all $j \in\{1, \ldots, n\}$. Assume that $Y$ is coarsely equivalent to $X$.

(i) Then there exists a separable Banach space $W$ such that $Y \oplus W$ is Lipschitz equivalent to $\bigoplus_{j=1}^{n}\left(X^{p_{j}} \oplus L_{p_{j}}\right)$.

(ii) Moreover, if $Y=Y^{p_{1}} \oplus \ldots \oplus Y^{p_{n}}$, where $Y^{p_{j}}$ is as. $\mathcal{L}_{p_{j}}$, for all $i \in\{1, \ldots, n\}$, then $\bigoplus_{j=1}^{n}\left(Y^{p_{j}} \oplus L_{p_{j}}\right)$ is Lipschitz equivalent to $\bigoplus_{j=1}^{n}\left(X^{p_{j}} \oplus L_{p_{j}}\right)$.

Proof. First we need some definitions. Let $\mathcal{U}$ be an ultrafilter on $\mathbb{N}$, and $Z$ be a Banach space. Then we define the ultrapower of $Z$ with respect to $\mathcal{U}$ as $Z_{\mathcal{U}}=$ $\left\{\left(z_{n}\right)_{n=1}^{\infty} \in Z^{\mathbb{N}} \mid \sup _{i \in \mathbb{N}}\left\|z_{n}\right\|<\infty\right\} / \sim$, where $\left(z_{n}\right)_{n=1}^{\infty} \sim\left(y_{n}\right)_{n=1}^{\infty}$ if $\lim _{n \in \mathcal{U}} \| z_{n}-$ $y_{n} \|=0 . Z_{\mathcal{U}}$ is a Banach space with norm $\left\|\left[\left(z_{n}\right)_{n=1}^{\infty}\right]\right\|=\lim _{n \in \mathcal{U}}\left\|z_{n}\right\|$, where $\left(z_{n}\right)_{n=1}^{\infty}$ is a representative of the class $\left[\left(z_{n}\right)_{n=1}^{\infty}\right] \in Z_{\mathcal{U}}$. Notice that $z \in Z \mapsto\left[(z)_{n=1}^{\infty}\right] \in Z_{\mathcal{U}}$ is a linear isometric embedding. If $Z$ is reflexive, $Z$ is 1 -complemented in the ultrapower $Z_{\mathcal{U}}$ (where the projection is given by $\left[\left(z_{n}\right)_{n}\right] \in Z_{\mathcal{U}} \mapsto w$ - $\lim _{n \in \mathcal{U}} z_{n} \in Z$ ), and we write $Z_{\mathcal{U}}=Z \oplus Z_{\mathcal{U}, 0}$. Also, we have that $(Z \oplus E)_{\mathcal{U}}=Z_{\mathcal{U}} \oplus E_{\mathcal{U}}$. We can now prove the lemma. For simplicity, let us assume that $n=2$.

(i) Let $\mathcal{U}$ be a nonprincipal ultrafilter on $\mathbb{N}$. As $Y$ is coarsely equivalent to $X$, $Y_{\mathcal{U}}$ is Lipschitz equivalent to $X_{\mathcal{U}}=X_{\mathcal{U}}^{p_{1}} \oplus X_{\mathcal{U}}^{p_{2}}$ (see [K], proposition 1.6). As the spaces $X_{\mathcal{U}}^{p_{j}}$ are reflexive, using the separable complementation property for reflexive spaces (see FJoP, Section 3), we can pick complemented separable subspaces $W \subset$ $Y_{\mathcal{U}, 0}$, and $X_{j, 0} \subset X_{\mathcal{U}, 0}^{p_{j}}$, for $j \in\{1,2\}$, such that $Y \oplus W$ is Lipschitz equivalent to $\left(X^{p_{1}} \oplus X_{1,0}\right) \oplus\left(X^{p_{2}} \oplus X_{2,0}\right)$. By enlarging $X_{j, 0}$ and $W$, if necessary, we can assume that $X_{j, 0}=L_{p_{j}}$, for $j \in\{1,2\}$ (this follows from Proposition 2.4.a of [JoLiS, Theorem I(ii) and Theorem III(b) of [LiRo ).

(ii) The same argument as why $X_{1,0} \oplus X_{2,0}$ can be enlarged so that $X_{1,0} \oplus X_{2,0}=$ $L_{p_{1}} \oplus L_{p_{2}}$ gives us that $W$ can also be assumed to be $L_{p_{1}} \oplus L_{p_{2}}$.

We can now prove Theorem 1.7. As mentioned in Section 1, Theorem 1.7 was proved in JoLiS (Theorem 5.8) for the cases $1<p_{1}<\ldots<p_{n}<2$ and $2<p_{1}<\ldots<p_{n}<\infty$. In our proof, Theorem [1.6 will play a similar role as Corollary 1.7 of [JoLiS did in their proof. Also, we use ideas in the proof of Theorem 5.3 of KRa in order to unify the cases $1<p_{1}<\ldots<p_{n}<2$ and $2<p_{1}<\ldots<p_{n}<\infty$. In order to avoid an unnecessarily extensive proof, we will only present the parts of the proof that require Theorem [1.6 above, and therefore 
are different from what can be found in the present literature.

Sketch of the proof of Theorem 1.7. By Proposition 5.7 of JoLiS, $T^{p}$ is uniformly homeomorphic to $T^{p} \oplus \ell_{p}$, for all $p \in[1, \infty)$. So, the backwards direction follows. Let us prove the forward direction. As uniform homeomorphism implies coarse equivalence, it is enough to assume that $Y$ is coarsely equivalent to $X$. By Theorem 1.6. $Y$ does not contain $\ell_{2}$. Let $m \in\{1, \ldots, n-1\}$ be such that $2 \in\left(p_{m}, p_{m+1}\right)$ (if such $m$ does not exist, the result simply follows from Theorem 5.8 of [JoLiS]).

Claim 1: $X \oplus \bigoplus_{j=1}^{n} L_{p_{j}}$ and $Y \oplus \bigoplus_{j=1}^{n} L_{p_{j}}$ are Lipschitz equivalent.

By Lemma $5.8(\mathrm{i})$, there exists a separable Banach space $W$ so that $Y \oplus W$ is Lipschitz equivalent to $\bigoplus_{j=1}^{n}\left(T^{p_{j}} \oplus L_{p_{j}}\right)$. Hence, the image of $Y$ through this Lipschitz equivalence is the range of a Lipschitz projection in $\bigoplus_{j=1}^{n}\left(T^{p_{j}} \oplus L_{p_{j}}\right)$. Therefore, by Theorem 2.2 of [HM], we have that $Y$ is isomorphic to a complemented subspace of $\bigoplus_{j=1}^{n}\left(T^{p_{j}} \oplus L_{p_{j}}\right)$. Let $A$ be this isomorphic embedding. For each $i \in\{m+1, \ldots, n\}$, let $\pi_{i}: Y \rightarrow L_{p_{i}}$ be the composition of $A$ with the projection $\bigoplus_{j=1}^{n}\left(T^{p_{j}} \oplus L_{p_{j}}\right) \rightarrow L_{p_{i}}$. As $Y$ does not contain $\ell_{2}, \pi_{i}$ factors through $\ell_{p_{i}}$ (see [Jo]). Hence, $Y$ is isomorphic to a complemented subspace of

$$
\bigoplus_{j=1}^{m}\left(T^{p_{j}} \oplus L_{p_{j}}\right) \oplus \bigoplus_{j=m+1}^{n}\left(T^{p_{j}} \oplus \ell_{p_{j}}\right) .
$$

As $Z_{1}:=\bigoplus_{j=1}^{m}\left(T^{p_{j}} \oplus L_{p_{j}}\right)$ and $Z_{2}:=\bigoplus_{j=m+1}^{n}\left(T^{p_{j}} \oplus \ell_{p_{j}}\right)$ are totally incomparable (i.e., none of their infinite dimensional subspaces are isomorphic), $Y \cong Y_{1} \oplus Y_{2}$, where $Y_{1}$ and $Y_{2}$ are complemented subspaces of $Z_{1}$ and $Z_{2}$, respectively (see [EW], Theorem 3.5). Hence, $Y_{1}^{*}$ is complemented in $Z_{1}^{*}$. Notice that, as $Y$ is coarsely equivalent to the super-reflexive space $X, Y$ is also super-reflexive (see [Ri], Theorem 1A). Hence, $Y_{1}$ is super-reflexive, and so is $Y_{1}^{*}$. As $Y_{1}$ has cotype 2 (see Remark 4.10) and $Y_{1}^{*}$ has non trivial type (as $Y_{1}^{*}$ is super-reflexive), it follows that $Y_{1}^{*}$ has type 2 (see the remark below Theorem 1 in $[\mathrm{P}]$ ). So, $Y_{1}^{*}$ does not contain a copy of $\ell_{2}$. Indeed, otherwise $Y_{1}^{*}$ would contain a complemented copy of $\ell_{2}$ (see [Ma]), contradicting that $Y_{1}$ does not contain a copy of $\ell_{2}$.

Proceeding similarly as above and using that $Y_{1}^{*}$ does not contain $\ell_{2}$, the main theorem of [J] implies that $Y_{1}^{*}$ is isomorphic to a complemented subspace of $\bigoplus_{j=1}^{m}\left(T^{p_{j}^{*}} \oplus \ell_{\tilde{p}_{j}}\right)$, where each $\tilde{p}_{j}$ is the conjugate of $p_{j}$ (i.e., $\left.1 / p_{j}+1 / \tilde{p}_{j}=1\right)$. Therefore, $Y_{1}$ embeds into $\bigoplus_{j=1}^{m}\left(T^{p_{j}} \oplus \ell_{p_{j}}\right)$ as a complemented subspace. This gives us that $Y$ embeds into $\bigoplus_{j=1}^{n}\left(T^{p_{j}} \oplus \ell_{p_{j}}\right)$ as a complemented subspace.

As the spaces $\left(T^{p_{j}} \oplus \ell_{p_{j}}\right)_{j=1}^{n}$ are totally incomparable, we can write $Y$ as $Y_{p_{1}} \oplus$ $\ldots \oplus Y_{p_{n}}$, where each $Y_{p_{j}}$ is a complemented subspace of $T^{p_{j}} \oplus \ell_{p_{j}}$ (see [EW], Theorem 3.5) and it is an as. $\mathcal{L}_{p_{j}}$ (see JoLiS, Lemma 2.5 and Proposition 2.7). By Lemma 5.8 (ii), we have that $X \oplus \bigoplus_{j=1}^{n} L_{p_{j}}$ and $Y \oplus \bigoplus_{j=1}^{n} L_{p_{j}}$ are Lipschitz equivalent.

Claim 2: There exists a quotient $W$ of $L_{p_{1}} \oplus \ldots \oplus L_{p_{n}}$ such that $Y \oplus W$ is isomorphic to $X \oplus \bigoplus_{j=1}^{n} L_{p_{j}}$. 
The prove of Claim 2 is the same as the proof of the claim in Proposition 2.10 of [JoLiS], so we do not present it here. Let us assume the claim and finish the proof. As $X$ does not contain any $\ell_{s}$, every operator of $X$ into $\oplus_{j=1}^{n} L_{p_{j}}$ is strictly singular (see KrMa, Theorem II.2 and Theorem IV.1). Therefore, by EW] (or [LiT, Theorem 2.c.13), $Y \cong Y_{X} \oplus Y_{L}$ and $W \cong W_{X} \oplus W_{L}$, where $Y_{X}$ and $W_{X}$ are complemented subspaces of $X, Y_{L}$ and $W_{L}$ are complemented subspaces of $\oplus_{j=1}^{n} L_{p_{j}}$, and $X \cong Y_{X} \oplus W_{X}$. Proceeding as in the proof of Claim 1 above, we get that $Y_{L}$ is complemented in $\oplus_{j=1}^{n} \ell_{p_{j}}$. So, $Y_{L}$ is either finite dimensional or isomorphic to $\oplus_{j \in F} \ell_{p_{j}}$, for some $F \subset\{1, \ldots, n\}$.

Let us show that $W_{X}$ is finite dimensional. Suppose this is not the case. As $W$ is a quotient of $\oplus_{j=1}^{n} L_{p_{j}}$, and $W_{X}$ is complemented in $W$, we have that $W_{X}^{*}$ embeds into $\oplus_{j=1}^{n} L_{\tilde{p}_{j}}$, where each $\tilde{p}_{j}$ is the conjugate of $p_{j}$. Therefore, it follows that $W_{X}^{*}$ must contain some $\ell_{s}$ (see $\mathrm{KrMa}$, Theorem II.2 and Theorem IV.1). As $W_{X}^{*}$ embeds into $X^{*}$, and $X^{*}$ does not contain any $\ell_{s}$, this gives us a contradiction.

As $X \cong Y_{X} \oplus W_{X}$, and $\operatorname{dim}\left(W_{X}\right)<\infty$, we have that $\operatorname{dim}\left(X / Y_{X}\right)<\infty$. Therefore, as $X$ is isomorphic to its hyperplanes, we conlude that $Y_{X} \cong X$. So, we are done.

Problem 5.9. Does Theorem 1.7 hold if $2 \in\left\{p_{1}, \ldots, p_{n}\right\}$ ?

Problem 5.10. What can we say if a Banach space $X$ is either coarsely or uniformly equivalent to the Tsirelson space $T$ ?

Remark 5.11. It is worth noticing that, using Remark 5.5 and adapting the proofs of Theorem 5.5 and Theorem 5.7 of KRa to our settings, one can show that $\left(\oplus T_{p}\right)_{T_{q}}$ does not coarse Lipschitz embed into $T_{p} \oplus T_{q}$, for all $p, q \in[1, \infty)$ with $p \neq q$.

Acknowledgments: I would like to thank my adviser C. Rosendal for all the help and attention he gave to this paper. I would also like to thank Th. Schlumprecht for helpful conversations about the results in Section 3 and the anonymous referee for their comments and suggestions.

\section{REFERENCES}

[A] I. Aharoni, Every separable metric space is Lipschitz equivalent to a subset of $c_{0}^{+}$, Israel J. Math. 19 (1974), 284-291.

[AlB] F. Albiac, and F. Baudier, Embeddability of snowflaked metrics with applications to the nonlinear geometry of the spaces $L_{p}$ and $\ell_{p}$ for $0<p<\infty$, J. Geom. Anal. 25 (2015), no. 1, $1-24$.

[B] F. Baudier, Quantitative nonlinear embeddings into Lebesgue sequence spaces, J. Topol. Anal. 8 (2016), no. 1, 117-150.

[BKL] F. Baudier, N. Kalton, and G. Lancien, A new metric invariant for Banach spaces, Studia Math. 199 (2010), 73-94.

[Be] B. Beauzamy, Banach-Saks properties and spreading models, Math. Scand. 44 (1979), no. 2, 357-384.

[BenLi] Y. Benyamini, and J. Lindenstrauss, Geometric nonlinear functional analysis. Vol. 1, American Mathematical Society Colloquium Publications, vol. 48, American Mathematical Society, Providence, RI, 2000.

[Br] B. M. Braga, On the complexity of some classes of Banach spaces and nonuniversality, Czech. Math. J. 64, no. 4 (2014), 1123-1147.

[BruSu] A. Brunel, and Sucheston, On J-convexity and ergodic super-properties of Banach spaces, Trans. Amer. Math. Soc. 204 (1975), 79-90.

[CSh] P. Casazza, and T. Shura, Tsirelson space, Lecture Notes in Mathematics, 1363, SpringerVerlag, Berlin, 1989. 
[CKKuM] P. G. Casazza, N. Kalton, D. Kutzarova, and M. Mastylo, Complex interpolation and complementably minimal spaces, 135-143, Interaction between functional analysis, harmonic analysis and probability, Dekker, 1996.

[D] N. Dew, Asymptotic Structure of Banach Spaces, Ph.D dissertation, University of Oxford, St. Johns College (2002).

[DiJT] J. Diestel, H. Jarchow, and A. Tong, Absolutely summing operators, Cambridge Studies in Advanced Mathematics 43, Cambridge University Press (1995).

[DimGoJ] V. Dimant, R. Gonzalo, and J. A. Jaramillo, Asympotic structure, $\ell_{p}$-estimates of sequences, and compactness of multilinear mappings, J. Math. Appli. 350 (2009) 680-693.

[Do] P. Dodos, Banach Spaces and Descriptive Set Theory: Selected Topics, Lecture Notes in Mathematics, Vol. 1993, Springer-Verlag, Berlin, 2010.

[EW] I. S. Edelstein, and P. P. Wojtaszczyk, On projections and unconditional bases in direct sums of Banach spaces, Studia Math. 56 (1976), 263-276.

[FJo] T. Figiel, and W. B. Johnson, A uniformly convex Banach space which contains no $\ell_{p}$, Compositio Math. 29 (1974), 179-190.

[FJoP] T. Figiel, W. Johnson, and A. PeÅĆczyÅĎski, Some approximation properties of Banach spaces and Banach lattices, Israel J. Math. 183 (2011), 199-231.

[GLZ] G. Godefroy, G. Lancien, and V. Zizler, The non-linear geometry of Banach spaces after Nigel Kalton, Rocky Mountain J. Math. 44 (2014), no. 5, 1529-1583.

[GuKa] E. Guentner, and J. Kaminker, Exactness and uniform embeddability of discrete groups, J. London Math. Soc. (2) 70 (2004), no. 3, 703-718.

$[\mathrm{HM}]$ S. Heinrich, and P. Mankiewicz, Applications of ultrapowers to the uniform and Lipschitz classification of Banach spaces, Studia Math. 73 (1982), 225-251.

[Jo] W. B. Johnson, Operators into $L_{p}$ which factor through $l_{p}$, J. London Math. Soc. 14 (1976), 333-339.

[Jo2] W. B. Johnson, A reflexive Banach space which is not sufficiently Euclidean, Studia Math. 55 (1976), 201-205.

[JoLiS] W. B. Johnson, J. Lindenstrass, and G. Schechtman, Banach spaces determined by their uniform structure, Geom. Funct. Anal. 6 (1996) 430-470.

[K] N. Kalton, The nonlinear geometry of Banach spaces, Rev. Mat. Complut. 21 (2008), 7-60.

[KRa] N. Kalton, and N. Randrianarivony, The coarse Lipschitz structure of $\ell_{p} \oplus \ell_{q}$, Math. Ann. 341 (2008), 223-237.

[KnOSh] H. Knaust, E. Odell, and Th. Schlumprecht, On asymptotic structure, the Szlenk index and UKK properties in Banach spaces, Positivity 3 (1999), no. 2, 173-199.

[KrMa] J. L. Krivine, and B. Maurey, Espaces de Banach stables, Israel J. Math. 39 (1981), 273-295.

[LiRo] J. Lindenstrauss, and H. Rosenthal, The $\mathcal{L}_{p}$ spaces, Israel J. Math. 71969 325-349.

[LiT] J. Lindenstrauss, and L. Tzafriri, Classical Banach Spaces, II, Function Spaces, Ergebnisse der Mathematik und ihrer Grenzgebiete [Results in Mathematics and Related Areas], Vol. (7, Springer-Verlag, Berlin, 1979.

[Ma] B. Maurey, Un theorème de prolongement, C. R. Acad., Paris 279 (1974), 329-332.

[MeN] M. Mendel, and A. Naor, Metric cotype, Ann. Math. (2) 168 (2008), no. 1, 247-298.

[R] M. Raja, On asymptotically uniformly smooth Banach spaces, J. Funct. Anal. 264 (2013), no. 2, 479-492.

[Ri] M. Ribe, On uniformly homeomorphic normed spaces, Ark. Math. 14 (1976), 237-244.

[Ro] H. P. Rosenthal, A Characterization of Banach Spaces Containing $\ell_{1}$, Proc. Nat. Acad. Sci. USA 71 (1974), no. 6, 2411-2413.

[OScZs] E. Odell, Th. Schlumprecht, and A. Zsák, On the structure of asymptotic $\ell_{p}$ spaces, Quart. J. Math. 59 (2008), 85-122.

[P] G. Pisier, On the duality between type and cotype, Martingale theory in harmonic analysis and Banach spaces (Cleveland, Ohio, 1981), pp. 131-144, Lecture Notes in Math., 939, Springer, Berlin, 1982.

[Sc] Th. Schlumprecht, An arbitrarily distortable Banach space, Israel J. Math. 76, 1991, 81-95.

\section{Appendix: COMputations For Remark 5.11}

In this appendix, we prove some technical results that will, in particular, gives us the claim in Remark 5.11 Precisely, we show the following. 
Theorem 6.1. Let $p, q \in[1, \infty)$, with $p \neq q$. Then $\left(\oplus T_{p}\right)_{T_{q}}$ does not coarse Lipschitz embed into $T_{p} \oplus T_{q}$.

Let $X$ be a sequence of Banach spaces, $\mathcal{E}=\left(e_{n}\right)_{n}$ be a 1-unconditional basic sequence, and consider the sum $(\oplus X)_{\mathcal{E}}$. We call a sequence $\left(x_{n}\right)_{n}$ in $(\oplus X)_{\mathcal{E}}$ a block sequence in $(\oplus X)_{\mathcal{E}}$ if there exists a strictly increasing sequence $\left(p_{n}\right)_{n} \in \mathbb{N}^{\mathbb{N}}$ such that $x_{n}=\sum_{j=p_{n}+1}^{p_{n+1}} y_{j}$, for all $n \in \mathbb{N}$, where $y_{p_{n}+1} \in X_{p_{n}+1}, \ldots, y_{p_{n+1}} \in X_{p_{n+1}}$. Notice that, if $\mathcal{E}$ is a weakly null sequence, then any bounded block sequence in $(\oplus X)_{\mathcal{E}}$ is weakly null. Indeed, this follows from the fact that a bounded sequence is weakly null if and only if all of its subsequences has a convex block subsequence converging to zero in norm (see, for example, $[\mathrm{Br}$, Lemma 19).

We omit the proof of the following two lemmas, as they are essentially the same as the proofs of Lemma 5.1 and Lemma 5.4 respectively.

Lemma 6.2. Let $\mathcal{E}$ be a weakly null 1-unconditional basis generating an asymptotically p-uniformly smooth Banach space, for some $p \in(1, \infty)$. Let $X$ be a Banach space. Let $x, y \in(\oplus X)_{\mathcal{E}}$, and $\delta>0$. Then, if $\left(x_{n}\right)_{n}$ is a block sequence in $B_{(\oplus X)_{\mathcal{E}}}$, there exists $n_{0} \in \mathbb{N}$, such that, for all $n>n_{0}$, we have

$$
u+\delta^{1 / p}\|v\| x_{n} \in \operatorname{Mid}(x, y, \delta),
$$

where $u=\frac{1}{2}(x+y)$, and $v=\frac{1}{2}(x-y)$.

Lemma 6.3. Let $1 \leq q<p$. Let $\mathcal{E}$ be a weakly null 1-unconditional basis generating an asymptotically $p$-uniformly smooth Banach space, for some $p \in(1, \infty)$. Let $X$ be a Banach space, and let $Y$ be a Banach space with a 1-unconditional basis satisfying a lower $\ell_{q}$-estimate with constant 1 . Let $f:(\oplus X)_{\mathcal{E}} \rightarrow Y$ be a coarse map. Then, for any $t>0$, and any $\delta \in(0,1)$, there exists $x \in(\oplus X)_{\mathcal{E}}$, a compact subset $K \subset Y$, and $\tau>t$, such that, for any block sequence $\left(x_{n}\right)_{n}$ in $B_{(\oplus X)_{\mathcal{E}}}$, there exists $n_{0} \in \mathbb{N}$ such that

$$
f\left(x+\tau x_{n}\right) \in K+\delta \tau B_{(\oplus X)_{\mathcal{E}}}, \quad \text { for all } n>n_{0} .
$$

Theorem 6.4. Let $1 \leq q<p$ and $1<r<l$. Assume that

(i) $X$ is a Banach space with the $r$-co-Banach-Saks property, with the $r$-BanachSaks property, and not containing $\ell_{1}$,

(ii) $\mathcal{E}$ is a weakly null normalized 1-unconditional basis generating an asymptotically p-uniformly smooth Banach space,

(iii) $Y_{1}$ is a Banach space with a 1-unconditional basis satisfying a lower $\ell_{q^{-}}$ estimate with constant 1 , and

(iv) $Y_{2}$ is a reflexive asymptotically l-uniformly smooth Banach space.

Then $(\oplus X)_{\mathcal{E}}$ does not coarse Lipschitz embed into $Y_{1} \oplus Y_{2}$.

Proof. Let $f=\left(f_{1}, f_{2}\right):(\oplus X)_{\mathcal{E}} \rightarrow Y_{1} \oplus_{1} Y_{2}$ be a coarse Lipschitz embedding. As $f$ is a coarse Lipschitz embedding, there exists $C>0$ such that $\rho_{f}(t) \geq C^{-1} t-C$, and $\omega_{f_{2}}(t) \leq C t+C$, for all $t>0$.

Fix $k \in \mathbb{N}$, and $\delta \in(0,1)$. By Lemma 6.3 , there exists $x \in(\oplus X)_{\mathcal{E}}, \tau>k$, and a compact subset $K \subset Y_{1}$ such that, for any block sequence $\left(y_{n}\right)_{n}$ in $B_{(\oplus X)}$, there exists $n_{0} \in \mathbb{N}$, such that 


$$
f_{1}\left(x+\tau y_{n}\right) \subset K+\delta \tau B_{Y_{1}}, \quad \text { for all } n>n_{0} .
$$

As $X$ does not contain $\ell_{1}$, there exists a normalized weakly null sequence $\left(x_{n}\right)_{n}$ in $X$, with $\inf _{n \neq m}\left\|x_{n}-x_{m}\right\|>0$. As $X$ has the $r$-Banach-Saks property, there exists $c>1$ (independent of $k$ ), such that, by taking a subsequence if necessary, we have that

$$
\left\|x_{n_{1}}+\ldots+x_{n_{k}}\right\| \leq c k^{1 / r}
$$

for all $n_{1}<\ldots<n_{k} \in \mathbb{N}$. For each $j \in \mathbb{N}$, denote by $x_{n}^{j}$ the element of $(\oplus X)_{\mathcal{E}}$ whose $j$-th $\mathcal{E}$-coordinate is $x_{n}$, and all other $\mathcal{E}$-coordinates are zero. Define $\varphi_{k, \delta}$ : $G_{k}(\mathbb{N}) \rightarrow(\oplus X)_{\mathcal{E}}$ as

$$
\varphi_{k, \delta}\left(n_{1}, \ldots, n_{k}\right)=x+\frac{\tau}{c} k^{-1 / r}\left(x_{n_{1}}^{n_{1}}+\ldots+x_{n_{k}}^{n_{1}}\right),
$$

for all $\left(n_{1}, \ldots, n_{k}\right) \in G_{k}(\mathbb{N})$. As $\operatorname{Lip}\left(f_{2} \circ \varphi_{k, \delta}\right) \leq 2 \tau k^{-1 / r} C c^{-1}+C$, by Theorem 3.3. there exists $\mathbb{M}_{k, \delta} \subset \mathbb{N}$ such that

$$
\operatorname{diam}\left(f_{2} \circ \varphi_{k, \delta}\left(G_{k}\left(\mathbb{M}_{k, \delta}\right)\right)\right) \leq 2 K \tau k^{1 / l-1 / r} C c^{-1}+K C k^{1 / l}
$$

for some $K>0$ independent of $k$ and $\delta$.

Similarly as in the proof of Theorem [5.6, by passing to a further subsequence if necessary, we have that, for all $\left(n_{1}, \ldots, n_{k}\right) \in G_{k}\left(\mathbb{M}_{k, \delta}\right)$,

$$
f_{1} \circ \varphi_{k, \delta}\left(n_{1}, \ldots, n_{k}\right) \subset K+\delta \tau B_{Y_{1}} .
$$

Therefore, by taking a further subsequence, we can assume that $\operatorname{diam}\left(f_{1} \circ \varphi_{k \delta}\left(\mathbb{M}_{k, \delta}\right)\right) \leq$ $3 \delta \tau$ (see Lemma 4.1 of [KRa]).

Without loss of generality, we may assume that $\mathbb{M}_{k+1, \delta} \subset \mathbb{M}_{k, \delta}$, for all $k \in \mathbb{N}$. Let $\mathbb{M}_{\delta} \subset \mathbb{N}$ be a diagonalization of $\left(\mathbb{M}_{k}\right)_{k}$. Using the fact that $X$ has the $r$-coBanach-Saks property to the weakly null sequence $\left(x_{n_{2 j-1}}-x_{n_{2 j}}\right)_{j} \in \mathbb{M}_{\delta}$, we get that, there exists $d>0$ (independent of $k$ ) such that, for all $k \in \mathbb{N}$, there exists $n_{1}<\ldots<n_{2 k} \in \mathbb{M}_{k, \delta}$, such that

$$
d k^{1 / r} \leq\left\|\sum_{j=1}^{k}\left(x_{n_{2 j-1}}-x_{n_{2 j}}\right)\right\| .
$$

Therefore, $\operatorname{diam}\left(f \circ \varphi_{k, \delta}\left(G_{k}\left(\mathbb{M}_{\delta}\right)\right)\right) \geq \tau d C^{-1} c^{-1}-C$, and we get that

$$
\tau d C^{-1} c^{-1}-C \leq 2 K \tau k^{1 / l-1 / r} C c^{-1}+3 C k^{1 / l}+K \delta \tau,
$$

for all $k \in \mathbb{N}$, and all $\delta \in(0,1)$. As $\tau>k$, we get that

$$
d C^{-1} c^{-1}-C k^{-1} \leq 2 K k^{1 / l-1 / r} C c^{-1}+K C k^{1 / l-1}+3 \delta,
$$


for all $k \in \mathbb{N}$, and all $\delta \in(0,1)$. As $l>r$, by letting $k \rightarrow \infty$ and $\delta \rightarrow 0$, we get a contradiction.

Theorem 6.5. Let $1 \leq q<r$ and $1<p<l$. Assume that

(i) $X$ is an asymptotically $r$-uniformly Banach space with the $r$-co-Banach-Saks property, and it does not contain $\ell_{1}$,

(ii) $\mathcal{E}$ be a weakly null normalized 1-unconditional basis with the p-co-Banach-Saks property, and the p-Banach-Saks property,

(iii) $Y_{1}$ be a Banach space with a 1-unconditional basis satisfying a lower $\ell_{q^{-}}$ estimate with constant 1 , and

(iv) $Y_{2}$ be a reflexive asymptotically l-uniformly smooth Banach space.

Then $(\oplus X)_{\mathcal{E}}$ does not coarse Lipschitz embed into $Y_{1} \oplus Y_{2}$.

Proof. Let $f=\left(f_{1}, f_{2}\right):(\oplus X)_{\mathcal{E}} \rightarrow Y_{1} \oplus_{1} Y_{2}$ be a coarse Lipschitz embedding. As $f$ is a coarse Lipschitz embedding, there exists $C>0$ such that $\rho_{f}(t) \geq C^{-1} t-C$, and $\omega_{f_{2}}(t) \leq C t+C$, for all $t>0$.

Fix $k \in \mathbb{N}$ and $\delta \in(0,1)$. As $X$ does not contain $\ell_{1}$, there exists a normalized weakly null sequence $\left(x_{n}\right)_{n}$ in $X$, with $\inf _{n \neq m}\left\|x_{n}-x_{m}\right\|>0$. For each $j \in \mathbb{N}$, denote by $x_{n}^{j}$ the element of $(\oplus X)_{\mathcal{E}}$ whose $j$-th $\mathcal{E}$-coordinate is $x_{n}$, and all other $\mathcal{E}$-coordinates are zero. As $\mathcal{E}$ has both the $p$-co-Banach-Saks property and the $p$ Banach-Saks property, by taking a subsequence of $\mathcal{E}$ if necessary, we can assume that

$$
d k^{1 / p} \leq\left\|\sum_{j=1}^{k}\left(x_{n_{2 j-1}}^{j}-x_{n_{2 j}}^{j}\right)\right\| \quad \text { and } \quad\left\|x_{n_{1}}^{1}+\ldots+x_{n_{k}}^{k}\right\| \leq c k^{1 / p},
$$

for all $n_{1}<\ldots<n_{2 k} \in \mathbb{N}$, where $c, d$ are positive constants (independent of $k$ ).

Let $\left(\oplus_{i=1}^{k} X\right)_{\mathcal{E}}$ be the subspace of $(\oplus X)_{\mathcal{E}}$ whose $j$-th coordinates are zero for all $j>k$, and define $\varphi: G_{k}(\mathbb{N}) \rightarrow\left(\oplus_{i=1}^{k} X\right)_{\mathcal{E}}$ as

$$
\varphi\left(n_{1}, \ldots, n_{k}\right)=x+\frac{\tau}{c} k^{-1 / p}\left(x_{n_{1}}^{1}+\ldots+x_{n_{k}}^{k}\right),
$$

for all $\left(n_{1}, \ldots, n_{k}\right) \in G_{k}(\mathbb{N})$.

As $\left(\oplus_{i=1}^{k} X\right)_{\ell_{\infty}}$ is asymptotically $p$-uniformly smooth, Lemma 5.4 and Remark 5.5 gives us that there exists $x \in\left(\oplus_{i=1}^{k} X\right)_{\mathcal{E}}, \tau>k$, and a compact subset $K \subset Y_{1}$ such that, for any weakly null sequence $\left(y_{n}\right)_{n}$ in $B_{\left(\oplus_{i=1}^{k} X\right)_{\mathcal{E}}}$, there exists $n_{0} \in \mathbb{N}$, such that

$$
f_{1}\left(x+\tau y_{n}\right) \subset K+\delta \tau B_{Y_{1}}, \quad \text { for all } n>n_{0} .
$$

So, similarly as in the proof of Theorem [5.6, there exists $\mathbb{M}_{0} \subset \mathbb{N}$ such that, for all $\left(n_{1}, \ldots, n_{k}\right) \in G_{k}\left(\mathbb{M}_{0}\right)$, we have

$$
f_{1} \circ \varphi\left(n_{1}, \ldots, n_{k}\right) \subset K+\delta \tau B_{Y_{1}} .
$$

Therefore, by taking a further subsequence $\mathbb{M}_{1} \subset \mathbb{M}_{0}$, can assume that $\operatorname{diam}\left(f_{1} \circ\right.$ $\left.\varphi\left(\mathbb{M}_{1}\right)\right) \leq 3 \delta \tau$. 
As $\operatorname{Lip}\left(f_{2} \circ \varphi\right) \leq 2 \tau k^{-1 / p} C c^{-1}+C$, by Theorem 3.3, there exists $\mathbb{M}_{2} \subset \mathbb{M}_{1}$ such that

$$
\operatorname{diam}\left(f_{2} \circ \varphi\left(G_{k}\left(\mathbb{M}_{2}\right)\right)\right) \leq 2 K \tau k^{1 / l-1 / p} C c^{-1}+K C k^{1 / l},
$$

for some $K>0$ independent of $k$ and $\delta$.

By Equation 6.1 we have that $\operatorname{diam}\left(f \circ \varphi\left(G_{k}\left(\mathbb{M}_{2}\right)\right)\right) \geq \tau d C^{-1} c^{-1}-C$, and we conclude that

$$
d C^{-1} c^{-1}-C k^{-1} \leq 2 K k^{1 / l-1 / p} C c^{-1}+K C k^{1 / l-1}+3 \delta,
$$

for all $\delta \in(0,1)$, and all $k \in \mathbb{N}$. As $l>p$, this gives us a contradiction.

Proof of Theorem 6.1. Theorem 6.4 gives us the case $p<q$, and Theorem 6.5 the case $q<p$.

Department of Mathematics, Statistics, and Computer Science (M/C 249), University of Illinois at Chicago, 851 S. Morgan St., Chicago, IL 60607-7045, USA

E-mail address: demendoncabraga@gmail.com 